\title{
Large Time Behavior of Solutions to Parabolic Equations with Dirichlet Operators and Nonlinear Dependence on Measure Data
}

\author{
Tomasz Klimsiak ${ }^{1,2}$ • Andrzej Rozkosz ${ }^{2}$
}

Received: 10 February 2017 / Accepted: 29 May 2018 / Published online: 8 June 2018

(C) The Author(s) 2018

\begin{abstract}
We study large time behavior of renormalized solutions of the Cauchy problem for equations of the form $\partial_{t} u-L u+\lambda u=f(x, u)+g(x, u) \cdot \mu$, where $L$ is the operator associated with a regular lower bounded semi-Dirichlet form $\mathcal{E}$ and $\mu$ is a nonnegative bounded smooth measure with respect to the capacity determined by $\mathcal{E}$. We show that under the monotonicity and some integrability assumptions on $f, g$ as well as some assumptions on the form $\mathcal{E}, u(t, x) \rightarrow v(x)$ as $t \rightarrow \infty$ for quasi-every $x$, where $v$ is a solution of some elliptic equation associated with our parabolic equation. We also provide the rate convergence. Some examples illustrating the utility of our general results are given.
\end{abstract}

Keywords Semilinear equation - Dirichlet operator - Mesure data $\cdot$ Large time behavior of solutions $\cdot$ Rate of convergence $\cdot$ Backward stochastic differential equation

Mathematics Subject Classification (2010) Primary: 35B40 - 35K58; Secondary: 60H30

\section{Introduction}

Let $E$ be a locally compact separable metric space, $m$ an everywhere dense Borel measure on $E$ and let $L$ be the operator associated with a regular lower bounded semi-Dirichlet

Tomasz Klimsiak

tomas@mat.uni.torun.pl

1 Institute of Mathematics, Polish Academy of Sciences, Śniadeckich 8, 00-956 Warszawa, Poland

2 Faculty of Mathematics and Computer Science, Nicolaus Copernicus University, Chopina 12/18, 87-100 Toruń, Poland 
form $(B, V)$ on $L^{2}(E ; m)$. The main purpose of the paper is to study large time behavior of solutions of the Cauchy problem

$$
\left\{\begin{array}{l}
\partial_{t} u-L u+\lambda u=f(x, u)+g(x, u) \cdot \mu \text { in }(0, \infty) \times E, \\
u(0, \cdot)=\varphi \text { on } E .
\end{array}\right.
$$

In Eq. 1.1, $\varphi: E \rightarrow \mathbb{R}, f, g: E \times \mathbb{R} \rightarrow \mathbb{R}$ are Borel measurable functions, $\mu$ is a smooth measure with respect to the parabolic capacity determined by $(B, V)$.

The class of operators corresponding to regular lower bounded Dirichlet forms is quite large. It contains both local operators whose model example is the Laplace operator $\Delta$ or Laplace operator perturbed by the first order operator, as well as nonlocal operators whose model example is the $\alpha$-Laplace operator $\Delta^{\alpha / 2}$ with $\alpha \in(0,2)$ or $\alpha$-Laplace operator with variable exponent $\alpha$ satisfying some regularity conditions. Many interesting examples of operators associated with regular semi-Dirichlet forms are to be found in $[11,15,18$, $22,26]$. In fact, our methods also allow to treat equations with operators associated with quasi-regular forms (see remarks at the end of Section 5).

As for the data $\varphi, f, g$, we assume that $\varphi \in L^{1}(E ; m), f, g$ are continuous and monotone in the second variable $u$ and satisfy mild integrability conditions. Our basic assumption on $\mu$ is that it is a smooth measure (with respect to the capacity associated with $(B, V)$ ) of class $\mathcal{R}^{+}(E)$, i.e. a positive smooth measure such that $E_{x} A_{\infty}^{\mu}<\infty$ for quasi-every (q.e. for short) $x \in E$, where $A^{\mu}$ is the additive functional of the Hunt process associated with $(B, V)$ in the Revuz correspondence with $\mu$. Equivalently, our condition imposed on $\mu$ means that the potential (associated with $(B, V))$ of $\mu$ is $m$-a.e. finite. It is known that if $(B, V)$ is a non-symmetric form, and moreover, it is transient or $\lambda>0$, then $\mathcal{R}^{+}(E)$ contains the class $\mathcal{M}_{0, b}^{+}(E)$ of positive bounded smooth measures on $E$ (see Section 2). In general, the inclusion $\mathcal{M}_{0, b}^{+}(E) \subset \mathcal{R}^{+}(E)$ is strict (see Section 2). Elliptic equations with unbounded measures of class $\mathcal{R}^{+}(E)$ are considered for instance in the monograph [23]; see also Section 6.

Let $v$ be a solution of the elliptic equation

$$
-L v+\lambda v=f(x, v)+g(x, v) \cdot \mu \quad \text { in } E .
$$

Our main result says that under the assumptions on $\varphi, f, g$ mentioned before and some additional mild assumptions on the semigroup $\left(P_{t}\right)$ and the resolvent $\left(R_{\alpha}\right)$ associated with $(B, V)$,

$$
\lim _{t \rightarrow \infty} u(t, x)=v(x)
$$

for q.e. $x \in E$. We also estimate the rate of convergence. Our main estimate says that for every $q \in(0,1)$ there is $C(q)>0$ such that for q.e. $x \in E$,

$$
|u(t, x)-v(x)| \leq 3 P_{t}|\varphi|(x)+3 P_{t}\left(R_{0}(|f(\cdot, 0)|+|g(\cdot, 0)| \cdot \tilde{\mu})\right)(x), \quad t>0 .
$$

The quantities on the right hand-side of Eq. 1.4 can be estimated for concrete operators $L$. We give some examples in Section 6.

To our knowledge, in case $L$ is a nonlocal operator, our results (1.3), (1.4) are entirely new. In case $L$ is local, we generalize the results obtained in the paper [12] in which $g \equiv 1$ and $L$ is a uniformly elliptic divergence form operator. Note, however, that in [12] systems of equations are treated. We also strengthen slightly the results of [20] concerning asymptotic behavior of nonnegative solutions of equations involving Laplace operator $\Delta$ and absorbing term of the form $h(u)|\nabla u|^{2}$ with $h$ satisfying the "sign condition". Some other results on asymptotic behavior, which are not covered by our approach, are to be found in [28-30]. In $[29,30]$ equations involving Leray-Lions type operators and smooth measure data are considered while [28] deals with linear equations with general, possibly singular, bounded 
measure $\mu$. Note that the methods used in [28-30] do not provide estimates between the parabolic solution and the corresponding stationary solution.

In order to prove (1.3) and (1.4), we develop the probabilistic approach initiated in [12]. We find interesting that it provides a unified way of treating a wide variety of seemingly disparate examples (see Section 6).

Although in the paper we deal mainly with the asymptotic behavior for solutions of Eq. 1.1, the first question we treat is the existence and uniqueness of solutions of problems (1.1) and (1.2). Here our results are also new, but our proofs rely on our earlier results proved in $[15,18]$ in case $g \equiv 1$. In fact, in the parabolic case we prove the existence and uniqueness of solutions to problems involving operators $L_{t}$ and data $f, g, \mu$ depending on time, i.e. more general then problem (1.1). Finally, let us note that in the paper we consider probabilistic solutions of Eqs. 1.2 and 1.3 (see Section 3 for the definitions). It is worth pointing out, however, that in the case where $\left(B^{(t)}, V\right)$ are (non-symmetric) Dirichlet forms, the probabilistic solutions coincide with the renormalized solutions defined in [17] (in the elliptic case under the additional assumption that $(B, V)$ satisfies the strong sector condition and either $(B, V)$ is transient or $\lambda>0)$. For local operators these renormalized solutions coincide with the usual renormalized solutions (see [9, 31] and also [16]).

\section{Preliminaries}

In the paper $E$ is a locally compact separable metric space, $E^{1}=\mathbb{R} \times E, m$ is an everywhere dense Borel measure on $E$ and $m_{1}=d t \otimes m$. For $T>0$ we write $E_{T}=[0, T] \times E$, $E_{0, T}=(0, T] \times E$. By $\mathcal{B}_{b}(E)$ we denote the set of all real bounded Borel measurable functions on $E$ and by $\mathcal{B}_{b}^{+}(E)$ we denote the subset of $\mathcal{B}_{b}(E)$ consisting of all nonnegative functions. The sets $\mathcal{B}_{b}\left(E^{1}\right), \mathcal{B}_{b}^{+}\left(E^{1}\right)$ are defined analogously.

\subsection{Dirichlet Forms}

Let $H=L^{2}(E ; m)$ and let $(\cdot, \cdot)$ denote the usual inner product in $H$. We assume that we are given a family $\left\{B^{(t)}, t \in[0, T]\right\}$ of regular semi-Dirichlet forms on $H$ with common domain $V \subset H$ (see [26, Section 1.1]). We assume that the forms $B^{(t)}$ are lower bounded and satisfy the sector condition with constants $\alpha_{0} \geq 0, K \geq 1$ independent of $t \in[0, T]$. Let us recall that this means that

$$
B_{\alpha_{0}}^{(t)}(\varphi, \varphi) \geq 0, \quad \varphi \in V
$$

where $B_{\lambda}^{(t)}(\varphi, \psi)=B^{(t)}(\varphi, \psi)+\lambda(\varphi, \psi)$ for $\lambda \geq 0$, and that

$$
\left|B_{\alpha_{0}}^{(t)}(\varphi, \psi)\right| \leq K B_{\alpha_{0}}^{(t)}(\varphi, \varphi)^{1 / 2} B_{\alpha_{0}}^{(t)}(\psi, \psi)^{1 / 2}, \quad \varphi, \psi \in V
$$

for all $t \in[0, T]$. Without loss of generality, we assume $\alpha_{0}<1$. We also assume that $[0, T] \ni t \mapsto B^{(t)}(\varphi, \psi)$ is Borel measurable for every $\varphi, \psi \in V$ and there is $c \geq 1$ such that

$$
c^{-1} B_{\alpha_{0}}(\varphi, \varphi) \leq B_{\alpha_{0}}^{(t)}(\varphi, \varphi) \leq c B_{\alpha_{0}}(\varphi, \varphi), \quad t \in[0, T], \varphi \in V,
$$

where $B(\varphi, \varphi)=B^{(0)}(\varphi, \varphi)$. By putting $B^{(t)}=B$ for $t \notin[0, T]$, we may and will assume that $B^{(t)}$ is defined and satisfies $(2.1)$ for all $t \in \mathbb{R}$. As usual, we denote by $\tilde{B}^{(t)}$ the symmetric part of $B^{(t)}$, i.e. $\tilde{B}^{(t)}(\varphi, \psi)=\frac{1}{2}\left(B^{(t)}(\varphi, \psi)+B^{(t)}(\psi, \varphi)\right)$.

Note that by the assumption, $V$ is a dense subspace of $H$ and the form $(B, V)$ is closed, i.e. $V$ is a real Hilbert space with respect to $\tilde{B}_{1}(\cdot, \cdot)$, which is densely and continuously 
embedded in $H$. We denote by $\|\cdot\|_{V}$ the norm in $V$, i.e. $\|\varphi\|_{V}^{2}=B_{1}(\varphi, \varphi), \varphi \in V$. We denote by $V^{\prime}$ the dual space of $V$, and by $\|\cdot\|_{V^{\prime}}$ the corresponding norm. We set $\mathcal{H}=L^{2}(\mathbb{R} ; H), \mathcal{V}=L^{2}(\mathbb{R} ; V), \mathcal{V}^{\prime}=L^{2}\left(\mathbb{R} ; V^{\prime}\right)$ and

$$
\|u\|_{\mathcal{V}}^{2}=\int_{\mathbb{R}}\|u(t)\|_{V}^{2} d t, \quad\|u\|_{\mathcal{V}^{\prime}}^{2}=\int_{\mathbb{R}}\|u(t)\|_{V^{\prime}}^{2} d t .
$$

We shall identify $H$ and its dual $H^{\prime}$. Then $V \subset H \simeq H^{\prime} \subset V^{\prime}$ continuously and densely, and hence $\mathcal{V} \subset \mathcal{H} \simeq \mathcal{H}^{\prime} \subset \mathcal{V}^{\prime}$ continuously and densely.

For $u \in \mathcal{V}$, we denote by $\frac{\partial u}{\partial t}$ the derivative in the distribution sense of the function $t \mapsto u(t) \in V$, and we set

$$
\mathcal{W}=\left\{u \in \mathcal{V}: \frac{\partial u}{\partial t} \in \mathcal{V}^{\prime}\right\}, \quad\|u\|_{\mathcal{W}}=\|u\|_{\mathcal{V}}+\left\|\frac{\partial u}{\partial t}\right\|_{\mathcal{V}^{\prime}} .
$$

We denote by $\mathcal{E}$ the time dependent Dirichlet form associated with the family $\left\{\left(B^{(t)}, V\right), t \in \mathbb{R}\right\}$, that is

$$
\mathcal{E}(u, v)= \begin{cases}\left\langle-\frac{\partial u}{\partial t}, v\right\rangle+\mathcal{B}(u, v), & u \in \mathcal{W}, v \in \mathcal{V}, \\ \left\langle\frac{\partial v}{\partial t}, u\right\rangle+\mathcal{B}(u, v), & u \in \mathcal{V}, v \in \mathcal{W},\end{cases}
$$

where $\langle\cdot, \cdot\rangle$ is the duality pairing between $\mathcal{V}^{\prime}$ and $\mathcal{V}$, and

$$
\mathcal{B}(u, v)=\int_{\mathbb{R}} B^{(t)}(u(t), v(t)) d t .
$$

Note that $\mathcal{E}$ can be identified with some generalized Dirichlet form (see [35, Example I.4.9(iii)]).

Given a time dependent form (2.4), we define quasi notions with respect to $\mathcal{E}$ (exceptional sets, nests, quasi-continuity as in [26, Section 6.2]. Note that by [26, Theorem 6.2.11] each element $u$ of $\mathcal{W}$ has a quasi-continuous $m_{1}$-version. We will denote it by $\tilde{u}$. Quasi-notions with respect to $(B, V)$ are defined as in [26, Section 2.2].

We denote by $S(E)$ the set of all smooth measures on $E$ with respect to the form $(B, V)$ (see, e.g., [26, Section 4.1] for the definition). $S\left(E^{1}\right)$ is the set of all smooth measures on $E^{1}$ with respect to $\mathcal{E}$ (see [14]), and $S\left(E_{0, T}\right)$ is the set of all smooth measures on $E^{1}$ with support in $E_{0, T}$. We denote by $\mathcal{M}_{b}\left(E_{0, T}\right)$ the set of all signed Borel measures on $E^{1}$ with support in $E_{0, T}$ such that $|\mu|\left(E^{1}\right)<\infty$, where $|\mu|$ stand for the total variation of $\mu . \mathcal{M}_{0, b}\left(E_{0, T}\right)$ (resp. $\mathcal{M}_{0, b}^{+}\left(E_{0, T}\right)$ ) is the subset of $\mathcal{M}_{b}\left(E_{0, T}\right)$ consisting of all smooth (resp. smooth nonnegative) measures. Analogously we define the classes $\mathcal{M}_{b}(E)$, $\mathcal{M}_{0, b}(E), \mathcal{M}_{0, b}^{+}(E)$.

We will say that a Borel measure $\mu$ on $E^{1}$ does not depend on time if it is of the form

$$
\mu=d t \otimes \tilde{\mu}
$$

for some Borel measure $\tilde{\mu}$ on $E$. Since $\tilde{\mu}(B)=\mu([0,1] \times B)$ for $B \in \mathcal{B}(E), \tilde{\mu}$ is uniquely determined by $\mu$. From now on, given $\mu$ not depending on time, we denote by $\tilde{\mu}$ the Borel measure on $E$ determined by Eq. 2.6.

Lemma 2.1 If $\mu \in S\left(E_{0, T}\right)$ does not depend on time, then $\tilde{\mu} \in S(E)$.

Proof Let $\alpha>\alpha_{0}$ and let Cap denote the capacity associated with the form $B_{\alpha}$ defined in [26, Definition 4 in Section 2.1], whereas CAP denote the capacity associated with $\mathcal{E}$ defined in [26, (6.2.18) in Section 6.2]. It is enough to prove that for every $A \subset E$, if $\operatorname{Cap}(A)=0$ then $\operatorname{CAP}([0, T] \times A)=0$. Suppose that $\operatorname{Cap}(A)=0$. Then by $[26, \mathrm{Eq}$. 
(2.1.8)], for every $\varepsilon>0$ there exists an open set $U_{\varepsilon} \subset E$ and $\psi_{\varepsilon} \in V$ such that $A \subset U_{\varepsilon}$, $\psi_{\varepsilon} \geq 1$ on $U_{\varepsilon}$ and

$$
B_{\alpha}\left(\psi_{\varepsilon}, \psi_{\varepsilon}\right) \leq \operatorname{Cap}\left(U_{\varepsilon}\right) \leq \varepsilon .
$$

By the above inequality and Eq. 2.1,

$$
B_{\alpha}^{(t)}\left(\psi_{\varepsilon}, \psi_{\varepsilon}\right) \leq c \varepsilon, \quad t \in \mathbb{R} .
$$

Let $f$ be a continuous function on $\mathbb{R}$ with compact support such that $f \geq 1$ on $[-T, 2 T]$ and let $\eta_{\varepsilon}=f \psi_{\varepsilon}$. Then $\eta_{\varepsilon} \in \mathcal{W}$ and by [26, (6.2.21)] and Eq. 2.7,

$$
\mathrm{CAP}([0, T] \times A) \leq C\left(\left\|\frac{\partial \eta_{\varepsilon}}{\partial t}\right\|_{L^{2}(0, T ; H)}^{2}+\mathcal{B}_{\alpha}\left(\eta_{\varepsilon}, \eta_{\varepsilon}\right)\right) \leq \varepsilon C^{\prime} T\left(\left\|\frac{\partial f}{\partial t}\right\|_{\infty}^{2}+\|f\|_{\infty}^{2}\right),
$$

where $C^{\prime}>0$ depends only on $c$ and $\alpha$. Since $\varepsilon>0$ was arbitrary, the desired result follows.

\subsection{Markov Processes and Additive Functionals}

In what follows $E \cup\{\partial\}$ is a one-point compactification of $E$. If $E$ is already compact then we adjoin $\partial$ to $E$ as an isolated point. When considering Dirichlet forms, we adopt the convention that every function $f$ on $E$ is extended to $E \cup\{\partial\}$ by setting $f(\partial)=0$. When considering time dependent Dirichlet forms, we adopt the convention that every function $\varphi$ on $E$ is extended to $E^{1}$ by setting $\varphi(t, x)=\varphi(x),(t, x) \in E^{1}$, and every function $f$ on $E^{1}$ (resp. $E_{0, T}$ ) is extended to $E^{1} \cup\{\partial\}$ by setting $f(\partial)=0$ (resp. $f(z)=0$ for $\left.z \in E^{1} \cup\{\partial\} \backslash E_{0, T}\right)$.

Let $\mathcal{E}$ be the form defined by Eq. 2.4. By [26, Theorem 6.3.1], there exists a Hunt process $\mathbf{M}=\left(\Omega,\left(\mathcal{F}_{t}\right)_{t \geq 0},\left(\mathbf{X}_{t}\right)_{t \geq 0},\left(P_{z}\right)_{z \in E^{1} \cup\{\partial\}}\right)$ with state space $E^{1}$, life time $\zeta$ and cemetery state $\partial$ associated with $\mathcal{E}$ in the resolvent sense, i.e. for every $\alpha>0$ and $f \in L^{2}\left(E^{1} ; m_{1}\right) \cap$ $\mathcal{B}_{b}\left(E^{1}\right)$ the resolvent of $\mathbf{M}$ defined as

$$
\mathbf{R}_{\alpha} f(z)=\int_{0}^{\infty} e^{-\alpha t} E_{z} f\left(\mathbf{X}_{t}\right) d t, \quad z \in E^{1}, f \in \mathcal{B}_{b}\left(E^{1}\right),
$$

is an $\mathcal{E}$-quasi-continuous $m_{1}$-version of the resolvent associated with the form $\mathcal{E}$. By [26, Theorem 6.3.1], if

$$
\mathbf{X}_{t}=\left(\tau(t), X_{\tau(t)}\right), \quad t \geq 0
$$

is a decomposition of $\mathbf{X}$ into the process on $\mathbb{R}$ and on $E$, then $\tau$ is the uniform motion to the right, i.e. $\tau(t)=\tau(0)+t, \tau(0)=s, P_{z}$-a.s. for $z=(s, x) \in E^{1}$. Moreover, one can check that if $B^{(t)}=B^{(0)}$ for $t \in \mathbb{R}$, then the process $\mathbb{M}^{(0)}=\left(\Omega,\left(\mathcal{F}_{t}\right)_{t \geq 0},\left(X_{t}\right)_{t \geq 0},\left(P_{0, x}\right)_{x \in E \cup\{\partial\}}\right)$ is a Hunt process with life time $\xi=\inf \left\{t \geq 0: X_{t} \in \partial\right\}$ associated with the form $\left(B^{(0)}, V\right)$.

Let us recall that an additive functional (AF for short) of $\mathbf{M}$ is called natural if $A$ and $\mathbf{M}$ have no common discontinuities. It is known (see [14, Section 2]) that for every $\mu \in S\left(E^{1}\right)$ there exists a unique positive natural $\mathrm{AF} A$ of $\mathbf{M}$ such that $A$ is in the Revuz correspondence with $\mu$, i.e. for every $m_{1}$-integrable $\alpha$-coexcessive function $h$ with $\alpha>0$,

$$
\lim _{\beta \rightarrow \infty} \beta E_{h \cdot m_{1}} \int_{0}^{\infty} e^{-(\alpha+\beta) t} f\left(\mathbf{X}_{t}\right) d A_{t}=\int_{E^{1}} f(z) h(z) \mu(d z), \quad f \in \mathcal{B}_{b}^{+}\left(E^{1}\right),
$$

where $E_{h \cdot m_{1}}$ denotes the expectation with respect to $P_{h \cdot m_{1}}(\cdot)=\int_{E^{1}} P_{z}(\cdot) h(z) m_{1}(d z)$. In what follows we will denote it by $A^{\mu}$. Conversely, if $A$ is a positive natural AF of $\mathbf{M}$ then modifying the proof of [11, Lemma 5.1.7] (we replace quasi-notions and facts used in the proof in [11] by the corresponding quasi-notions and facts from [26, Sections 2-4]; for the 
case of (non-symmetric) Dirichlet form see also [25, Theorem 5.6]) one can show that there exists a smooth measure on $E^{1}$ such that $A$ is in the Revuz correspondence with $\mu$.

We set

$$
\mathcal{R}\left(E_{0, T}\right)=\left\{\mu:|\mu| \in S\left(E_{0, T}\right), E_{z} \int_{0}^{\zeta_{\tau}} d A_{t}^{|\mu|}<\infty \text { for } m_{1} \text {-a.e. } z \in E_{0, T}\right\},
$$

where

$$
\zeta_{\tau}=\zeta \wedge(T-\tau(0)) .
$$

By [14, Proposition 3.4], in the definition of $\mathcal{R}\left(E_{0, T}\right)$ one can replace $m_{1}$-a.e. by q.e. (with respect to $\mathcal{E}$ ). By [14, Proposition 3.8], if $(B, V)$ is a (non-symmetric) Dirichlet form or, more generally, a semi-Dirichlet form satisfying the duality condition (see [14] for the definition), then $\mathcal{M}_{0, b}\left(E_{0, T}\right) \subset \mathcal{R}\left(E_{0, T}\right)$. The inclusion may be strict (see [14, Example 5.2]).

Let $\mu \in S(E)$. Since $\mathbb{M}^{(0)}$ corresponds to $(B, V)$, by [26, Theorem 4.1.16] there is a unique positive continuous $\mathrm{AF} A^{0, \mu}$ of $\mathbb{M}^{(0)}$ such that $A^{0, \mu}$ is in the Revuz correspondence with $\mu$, i.e.

$$
\lim _{\alpha \rightarrow \infty} \alpha E_{m} \int_{0}^{\infty} e^{-\alpha t} f\left(X_{t}\right) d A_{t}^{0, \mu}=\int_{E} f(x) \mu(d x), \quad f \in \mathcal{B}_{b}^{+}(E) .
$$

We set

$$
\mathcal{R}(E)=\left\{\mu:|\mu| \in S(E), E_{0, x} \int_{0}^{\zeta} d A_{t}^{0,|\mu|}<\infty \text { for } m \text {-a.e. } x \in E\right\} .
$$

By [15, Lemma 4.2], in the above definition of the class $\mathcal{R}(E)$ one can replace $m$-a.e. by q.e. (with respect to $(B, V)$ ), and by [18, Proposition 3.2], if $(B, V)$ is a transient (nonsymmetric) Dirichlet form, then $\mathcal{M}_{0, b}(E) \subset \mathcal{R}(E)$. In general, the inclusion is strict (see remarks following [18, Proposition 3.2]).

While considering elliptic equations and large time behavior of parabolic equations, we will assume that

$$
B^{(t)}(\varphi, \psi)=B(\varphi, \psi), \quad \varphi, \psi \in V, \quad t \in \mathbb{R}
$$

\section{Lemma 2.2 Assume (2.9).}

(i) For every $s \geq 0$ the distribution of $\left(X \circ \theta_{\tau(0)}, A^{0, \tilde{\mu}} \circ \theta_{\tau(0)}\right)$ under $P_{s, x}$ is equal to the distribution of $\left(X, A^{0, \tilde{\mu}}\right)$ under $P_{0, x}$.

(ii) $A^{\mu}=A^{0, \tilde{\mu}} \circ \theta_{\tau(0)}$.

Proof (i) We first suppose that $\tilde{\mu}(d x)=f(x) m(d x)$ for some $f \in L^{1}(E ; m)$. Then $A_{t}^{0, \tilde{\mu}}=$ $\int_{0}^{t} f\left(X_{r}\right) d r$, and hence $A_{t}^{0, \tilde{\mu}} \circ \theta_{\tau(0)}=\int_{0}^{t} f\left(X_{r} \circ \theta_{\tau(0)}\right) d r$. Therefore (i) follows from the fact that the distribution of $X$ under $P_{0, x}$ is equal to the distribution of $X \circ \theta_{\tau(0)}$ under $P_{s, x}$. Now assume that $\mu$ belongs to the set $S_{0}(E)$ of smooth measures of finite energy. Then $A_{t}^{0, \tilde{\mu}}=\int_{0}^{t} e^{r} d \tilde{A}_{r}$, where $\tilde{A}_{t}=\lim _{n \rightarrow \infty} \tilde{A}_{t}^{n}$ and $\tilde{A}_{t}^{n}=\int_{0}^{t} e^{-r} f_{n}\left(X_{r}\right) d r$ for some $f_{n} \in L^{1}(E ; m)$ (see the proof of [11, Theorem 5.1.1] or [26, Theorem 4.1.10]). From this and the first part we deduce that (i) is satisfied for every $\tilde{\mu} \in S_{0}(E)$. By [26, Lemma 4.1.14], there exists a nest $\left\{F_{n}\right\}$ such that $\mathbf{1}_{F_{n}} \cdot \tilde{\mu} \in S_{0}(E)$ for each $n \in \mathbb{N}$. Since we already know that (i) holds for $\tilde{\mu}$ replaced by $\mathbf{1}_{F_{n}} \cdot \tilde{\mu}$, applying the monotone convergence theorem we conclude that it holds for $\tilde{\mu}$ replaced by $\mathbf{1}_{\cup_{n=1}^{\infty} F_{n}} \cdot \tilde{\mu}$, and hence for $\tilde{\mu}$ because the set $E \backslash \bigcup_{n=1}^{\infty} F_{n}$ is exceptional. 
(ii) Let $A=A^{0, \tilde{\mu}} \circ \theta_{\tau(0)}$. Under (2.9) the distribution of $A$ under $P_{s, x}$ is equal to the distribution of $A^{0, \tilde{\mu}}$ under $P_{0, x}$. Hence

$$
E_{s, x} \int_{0}^{\infty} e^{-\alpha t} d A_{t}=E_{s, x} \int_{0}^{\infty} e^{-\alpha t} d\left(A_{t}^{0, \tilde{\mu}} \circ \theta_{s}\right)=E_{0, x} \int_{0}^{\infty} e^{-\alpha t} d A_{t}^{0, \tilde{\mu}}=: R_{\alpha} \tilde{\mu}(x) .
$$

One can check that $A$ is a CAF of M. Let $v$ denote its Revuz measure. Then for every $f$ of the form $f=\xi g$ with $\xi \in \mathcal{B}_{b}^{+}(\mathbb{R}), g \in \mathcal{B}_{b}^{+}(E)$ we have

$$
\begin{aligned}
\int_{E^{1}} f(z) v(d z) & =\lim _{\alpha \rightarrow \infty} \alpha \int_{E^{1}}\left(f(z) E_{z} \int_{0}^{\infty} e^{-\alpha t} d A_{t}\right) m_{1}(d z) \\
& =\lim _{\alpha \rightarrow \infty} \alpha \int_{E^{1}} \xi(s) g(x) R_{\alpha} \tilde{\mu}(x) d s m(d x) \\
& =\int_{\mathbb{R}} \xi(s) d s \cdot \int_{E} g(x) \tilde{\mu}(d x)=\int_{E^{1}} f(s, x) d s \tilde{\mu}(d x) .
\end{aligned}
$$

Hence $v=d t \otimes \tilde{\mu}=\mu$. Since additive functionals are uniquely determined by their Revuz measures, this proves (ii).

\section{Parabolic PDEs and Generalized BSDEs}

For $t \in[0, T]$ let $L_{t}$ denote the operator associated with the form $\left(B^{(t)}, V\right)$, i.e.

$$
D\left(L_{t}\right)=\left\{u \in V: v \mapsto B^{(t)}(u, v) \text { is continuous with respect to }(\cdot, \cdot)_{H}^{1 / 2} \text { on } V\right\}
$$

and

$$
\left(-L_{t} \varphi, \psi\right)=B^{(t)}(\varphi, \psi), \quad \varphi \in D\left(L_{t}\right), \psi \in V
$$

(see [22, Proposition I.2.16]). Suppose we are given measurable functions $\varphi: E \rightarrow \mathbb{R}$, $f, g: E_{T} \times \mathbb{R} \rightarrow \mathbb{R}$ and $\mu \in \mathcal{R}\left(E_{0, T}\right)$. In this section we consider the following Cauchy problems with terminal and initial conditions:

$$
\partial_{t} u+L_{t} u=-f(t, x, u)-g(t, x, u) \cdot \mu, \quad u(T)=\varphi
$$

and

$$
\partial_{t} u-L_{t} u=f(t, x, u)+g(t, x, u) \cdot \mu, \quad u(0)=\varphi .
$$

Definition Let $z \in E_{T}$. We say that a pair $\left(Y^{z}, M^{z}\right)$ is a solution of the BSDE

$$
Y_{t}^{z}=\varphi\left(\mathbf{X}_{\zeta_{\tau}}\right)+\int_{t \wedge \zeta_{\tau}}^{\zeta_{\tau}} f\left(\mathbf{X}_{r}, Y_{r}^{z}\right) d r+\int_{t \wedge \zeta_{\tau}}^{\zeta_{\tau}} g\left(\mathbf{X}_{r}, Y_{r}^{z}\right) d A_{r}^{\mu}-\int_{t \wedge \zeta_{\tau}}^{\zeta_{\tau}} d M_{r}^{z}, \quad t \geq 0,
$$

on the space $\left(\Omega, \mathcal{F}, P_{z}\right)$ if

(a) $Y^{z}$ is an $\left(\mathcal{F}_{t}\right)$-progressively measurable process of class D under $P_{z}, M^{z}$ is an $\left(\mathcal{F}_{t}\right)$ martingale under $P_{z}$ such that $M_{0}^{z}=0$,

(b) $\int_{0}^{\zeta_{\tau}}\left|f\left(\mathbf{X}_{t}, Y_{t}^{z}\right)\right| d t<\infty, \int_{0}^{\zeta_{\tau}}\left|g\left(\mathbf{X}_{t}, Y_{t}^{z}\right)\right| d\left|A^{\mu}\right|_{t}<\infty, P_{z}$-a.s. (Here $\left|A^{\mu}\right|_{t}$ denotes the total variation of the process $A^{\mu}$ on $[0, t]$ ),

(c) Eq. 3.4 is satisfied $P_{z}$-a.s.

Let us recall that a càdlàg $\left(\mathcal{F}_{t}\right)$-adapted process $Y$ is of Doob's class $\mathrm{D}$ under $P_{z}$ if the collection $\left\{Y_{\tau}: \tau \in \mathcal{T}\right\}$, where $\mathcal{T}$ is the set of all finite valued $\left(\mathcal{F}_{t}\right)$-stopping times, is 
uniformy integrable under $P_{z}$. Let $\mathcal{L}^{1}\left(P_{z}\right)$ denote the space of càdlàg $\left(\mathcal{F}_{t}\right)$-adapted processes $Y$ with finite norm

$$
\|Y\|_{z, 1}=\sup \left\{E_{z}\left|Y_{\tau}\right|: \tau \in \mathcal{T}\right\}
$$

It is known that $\mathcal{L}^{1}\left(P_{z}\right)$ is complete (see [10, p. 90]). Moreover, if processes $Y^{n}$ are of class $\mathrm{D}$ and $Y^{n} \rightarrow Y$ in $\mathcal{L}^{1}\left(P_{z}\right)$, then $Y$ is of class D. To see this, let us fix $\varepsilon>0$ and choose $n$ so that $\left\|Y^{n}-Y\right\|_{z, 1} \leq \varepsilon / 2$. Since the family $\left\{Y_{\tau}^{n}\right\}$ is of class $\mathrm{D}$, there exists $\delta>0$ such that if $P_{z}(A)<\delta$, then $\int_{A}\left|Y_{\tau}^{n}\right| d P_{z}<\varepsilon / 2$. It follows that if $P_{z}(A)<\delta$ then for every finite $\left(\mathcal{F}_{t}\right)$-stopping time $\tau$,

$$
\int_{A}\left|Y_{\tau}\right| d P_{z} \leq E_{z}\left|Y_{\tau}^{n}-Y_{\tau}\right|+\int_{A}\left|Y_{\tau}^{n}\right| d P_{z} \leq \varepsilon,
$$

which shows that $\left\{Y_{\tau}\right\}$ is uniformly integrable (see [34, Theorem I.11]).

To simplify notation, in what follows we write

$$
f_{u}(t, x):=f(t, x, u(t, x)), \quad g_{u}(t, x):=g(t, x, u(t, x)) .
$$

Definition (a) We say that $u: E_{0, T} \rightarrow \mathbb{R}$ is a solution of problem (3.2) if $f_{u} \cdot m \in \mathcal{R}\left(E_{0, T}\right)$, $g_{u} \cdot \mu \in \mathcal{R}\left(E_{0, T}\right)$ and for q.e. $z \in E_{0, T}$,

$$
u(z)=E_{z}\left(\varphi\left(\mathbf{X}_{\zeta_{\tau}}\right)+\int_{0}^{\zeta_{\tau}} f_{u}\left(\mathbf{X}_{t}\right) d t+\int_{0}^{\zeta_{\tau}} g_{u}\left(\mathbf{X}_{t}\right) d A_{t}^{\mu}\right) .
$$

(b) We say that $u:[0, T) \times E \rightarrow \mathbb{R}$ is a solution of problem (3.3) if $\bar{u}$ defined as

$$
\bar{u}(t, x):=u(T-t, x), \quad(t, x) \in E_{0, T},
$$

is a solution of the Cauchy problem with terminal condition of the form

$$
\partial_{t} \bar{u}+L_{T-t} \bar{u}=-f(T-t, x, \bar{u})-g(T-t, x, \bar{u}) \cdot\left(\mu \circ \iota_{T}^{-1}\right), \quad \bar{u}(T)=\varphi,
$$

where $\iota_{T}: E_{T} \rightarrow E_{T}, \iota_{T}(t, x)=(T-t, x)$.

Remark 3.1 If Eq. 3.6 has the uniqueness property (i.e. has a unique solution $v_{T}$ for every $T>0$ ), then for every $a>0$,

$$
\bar{v}_{T}(t, x)=v_{T}(T-t, x)=v_{T+a}(T+a-t, x)=\bar{v}_{T+a}(t, x), \quad(t, x) \in[0, T) \times E .
$$

To see this, let us write $f_{v_{T}}^{T}(x, t):=f\left(T-t, x, v_{T}(t, x)\right), g_{v_{T}}^{T}(x, t):=f(T-$ $\left.t, x, v_{T}(t, x)\right)$. With this notation,

$$
\frac{\partial v_{T}}{\partial t}+L_{T-t} v_{T}=f_{v_{T}}^{T}+g_{v_{T}}^{T} \cdot\left(\mu \circ \iota_{T}^{-1}\right), \quad v_{T}(T)=\varphi
$$

and

$$
\frac{\partial v_{T+a}}{\partial t}+L_{T+a-t} v_{T+a}=f_{v_{T+a}}^{T+a}+g_{v_{T+a}}^{T+a} \cdot\left(\mu \circ \iota_{T+a}^{-1}\right), \quad v_{T+a}(T+a)=\varphi .
$$

Of course, Eq. 3.7 will be proved once we show that

$$
v_{T}(t, x)=v_{T+a}(a+t, x), \quad(t, x) \in E_{0, T} .
$$


It is known (see [14, p. 1213]) that there exists a generalized nest $\left\{F_{n}\right\}$ on $E_{0, T+a}$ such that $\Phi^{n, T+a}:=\mathbf{1}_{F_{n}} \cdot\left(f_{v_{T+a}}^{T+a}+g_{v_{T+a}^{T+a}}^{T+a} \cdot\left(\mu \circ \iota_{T+a}^{-1}\right)\right) \in S_{0}\left(E_{0, T+a}\right)$ for each $n \in \mathbb{N}$. Let $v_{T+a}^{n}$ denote the solution of the linear equation

$$
\frac{\partial v_{T+a}^{n}}{\partial t}+L_{T+a-t} v_{T+a}^{n}=\Phi^{n, T+a}, \quad v_{T+a}^{n}(T+a)=\varphi,
$$

and let

$$
v_{T+a, a}^{n}(t, x):=v_{T+a}^{n}(a+t, x), \quad(t, x) \in(-a, T] \times E .
$$

By [14, Theorem 3.7], $v_{T+a}^{n}$ is a weak solution of Eq. 3.10. Therefore making a simple change of variables shows that $v_{T+a, a}^{n}$ is a weak solution of the linear equation

$$
\frac{\partial v_{T+a, a}^{n}}{\partial t}+L_{T-t} v_{T+a, a}^{n}=\mathbf{1}_{F_{n}}^{a} \cdot\left(f_{v_{T+a, a}}^{T}+g_{v_{T+a, a}^{T}}^{T} \cdot\left(\mu \circ \iota_{T}^{-1}\right)\right), \quad v_{T+a, a}^{n}(T)=\varphi,
$$

where $\mathbf{1}_{F_{n}}^{a}(t, x)=\mathbf{1}_{F_{n}}(t+a, x)$. Using the probabilistic representation of the solution of Eq. 3.10 and the fact that $\left\{F_{n}\right\}$ is a nest, one can easily show that $v_{T+a}^{n} \rightarrow v_{T+a}$ pointwise as $n \rightarrow \infty$. Similarly, using the probabilistic representation of the solution of Eq. 3.12 one can show that $v_{T+a, a}^{n}$ converges pointwise as $n \rightarrow \infty$ to the solution of Eq. 3.8, that is to $v_{T}$. This and Eq. 3.11 imply (3.9).

In the rest of this section we say that some property is satisfied quasi-everywhere (q.e. for brevity) if the set of those $z \in E^{1}$ for which it does not hold is exceptional with respect to the form $\mathcal{E}$.

In what follows we say that a Borel measurable $F: E_{0, T} \rightarrow \mathbb{R}$ is $\mu$-quasi-integrable $\left(F \in q L^{1}\left(E_{0, T} ; \mu\right)\right.$ in notation) if $P_{z}\left(\int_{0}^{\zeta_{\tau}}\left|F\left(\mathbf{X}_{t}\right)\right| d A_{t}^{\mu}<\infty\right)=1$ for q.e. $z \in E_{0, T}$.

Let us remark that if $\mu=m_{1}$, then $A_{t}^{\mu}=t, t \geq 0$, so $m_{1}$-quasi-integrability coincides with the notion of quasi-integrability considered in [14, Section 5]) (see also [13, Section 2]).

Our basic assumptions on the data are the following.

(P1) $\varphi \in L^{1}(E ; m), \mu \in \mathcal{R}^{+}\left(E_{0, T}\right)$.

(P2) $f(\cdot, \cdot, y), g(\cdot, \cdot, y)$ are measurable for every $y \in \mathbb{R}$ and $f(t, x, \cdot), g(t, x, \cdot)$ are continuous for every $(t, x) \in E_{0, T}$.

(P3) There is $\alpha \in \mathbb{R}$ such that $\left(f(t, x, y)-f\left(t, x, y^{\prime}\right)\right)\left(y-y^{\prime}\right) \leq \alpha\left|y-y^{\prime}\right|^{2}$ for all $y, y^{\prime} \in \mathbb{R}$ and $(t, x) \in E_{0, T}$.

(P4) $f(\cdot, \cdot, 0) \cdot m_{1} \in \mathcal{R}\left(E_{0, T}\right)$ and $(t, x) \mapsto f(t, x, y) \in q L^{1}\left(E_{0, T} ; m_{1}\right)$ for every $y \in \mathbb{R}$.

(P5) $\left(g(t, x, y)-g\left(t, x, y^{\prime}\right)\right)\left(y-y^{\prime}\right) \leq 0$ for all $y, y^{\prime} \in \mathbb{R}$ and $(t, x) \in E_{0, T}$.

(P6) $g(\cdot, \cdot, 0) \cdot \mu \in \mathcal{R}\left(E_{0, T}\right)$ and $(t, x) \mapsto g(t, x, y) \in q L^{1}\left(E_{0, T} ; \mu\right)$ for every $y \in \mathbb{R}$.

In what follows we denote by $\mathcal{D}^{q}\left(P_{z}\right), q>0$, the space of all $\left(\mathcal{F}_{t}\right)$-progressively measurable càdlàg processes $Y$ such that $E_{z} \sup _{t \geq 0}\left|Y_{t}\right|^{q}<\infty$.

Theorem 3.2 Assume that (P1)-(P6) are satisfied and $A^{\mu}$ is continuous.

(i) There exists a unique solution $u$ of problem (3.2).

(ii) Let

$$
M_{t}^{z}=E_{z}\left(\varphi\left(\mathbf{X}_{\zeta_{\tau}}\right)+\int_{0}^{\zeta_{\tau}} f_{u}\left(\mathbf{X}_{s}\right) d s+\int_{0}^{\zeta_{\tau}} g_{u}\left(\mathbf{X}_{s}\right) d A_{s}^{\mu} \mid \mathcal{F}_{t}\right)-u\left(\mathbf{X}_{0}\right) .
$$

Then there exists a càdlàg $\left(\mathcal{F}_{t}\right)$-adapted process $M$ such that $M_{t}=M_{t}^{z}, t \in[0, T]$, $P_{z}$-a.s. for q.e. $z \in E_{0, T}$, and for q.e $z \in E_{0, T}$ the pair $(u(\mathbf{X}), M)$ is a unique solution 
of Eq. 3.4 on the space $\left(\Omega, \mathcal{F}, P_{z}\right)$. Moreover, $u(\mathbf{X}) \in \mathcal{D}^{q}\left(P_{z}\right)$ for $q \in(0,1)$ and $M$ is a uniformly integrable martingale under $P_{z}$ for q.e. $z \in E_{0, T}$. Finally, for q.e. $z \in E_{0, T}$,

$$
\begin{aligned}
& E_{z} \int_{0}^{\zeta_{\tau}} f_{u}\left(\mathbf{X}_{t}\right) d t+\int_{0}^{\zeta_{\tau}} g_{u}\left(\mathbf{X}_{t}\right) d A_{t}^{\mu} \\
& \quad \leq E_{z}\left(\varphi\left(\mathbf{X}_{\zeta_{\tau}}\right)+2 \int_{0}^{\zeta_{\tau}}\left|f\left(\mathbf{X}_{t}, 0\right)\right| d t+3 \int_{0}^{\zeta_{\tau}}\left|g\left(\mathbf{X}_{t}, 0\right)\right| d A_{t}^{\mu}\right)
\end{aligned}
$$

Proof By using the standard change of variables (see, e.g., the beginning of the proof of [4, Lemma 3.1]), without loss of generality we may and will assume that $\alpha \leq 0$ in condition (P3).

We first prove (ii). The uniqueness of a solution of BSDE (3.4) follows from (P3), (P5) and the fact that $\mu$ is nonnegative. The proof is standard. We may argue for instance as in the proof of [15, Proposition 2.1] with obvious changes. We divide the proof of existence of a solution into two steps.

Step 1. Let $\xi=\varphi\left(\mathbf{X}_{\zeta_{\tau}}\right), f(t, y)=f\left(\mathbf{X}_{t}, y\right), g(t, y)=g\left(\mathbf{X}_{t}, y\right)$ and let $A$ be a continuous increasing $\left(\mathcal{F}_{t}\right)$-adapted process. Assume that

$$
T \cdot \sup _{0 \leq t \leq T}|f(t, 0)|+A_{T} \cdot \sup _{0 \leq t \leq T}|g(t, 0)|+|\xi| \leq c
$$

$P_{z}$-a.s. $z \in E_{0, T}$ for some $c>0$, and write $\bar{f}_{c}(t, y)=f\left(t, T_{c}(y)\right), \bar{g}_{c}(t, y)=g\left(t, T_{c}(y)\right)$, where

$$
T_{c}(y)=((-c) \vee y) \wedge c, \quad y \in \mathbb{R} .
$$

Then modifying slightly the proof of [15, Lemma 2.6], we show that there exists a unique solution $(Y, M)$ of the BSDE

$$
\begin{aligned}
Y_{t}=\xi & +\int_{t \wedge \zeta_{\tau}}^{\zeta_{\tau}} \bar{f}_{c}\left(s, Y_{s}\right) d s+\int_{t \wedge \zeta_{\tau}}^{\zeta_{\tau}}\left(\bar{g}_{c}\left(s, Y_{s}\right)-g(s, 0)\right) d A_{s}^{\mu} \\
& +\int_{t \wedge \zeta_{\tau}}^{\zeta_{\tau}} \bar{g}_{c}(s, 0) d A_{s}-\int_{t \wedge \zeta_{\tau}}^{\zeta_{\tau}} d M_{s}, \quad t \geq 0,
\end{aligned}
$$

on the space $\left(\Omega, \mathcal{F}, P_{z}\right)$ (for brevity, in our notation we drop the dependence of $Y, M$ on $z$ ). Let $\operatorname{sgn}(x)=1$ if $x>0$ and $\operatorname{sgn}(x)=-1$ if $x \leq 0$. By the Meyer-Tanaka formula (see [34, p. 216]) and the fact that $A^{\mu}$ is continuous,

$$
\begin{aligned}
\left|Y_{t}\right| \leq\left|Y_{\zeta_{\tau}}\right| & -\int_{t \wedge \zeta_{\tau}}^{\zeta_{\tau}} \operatorname{sgn}\left(Y_{s}\right) d Y_{s} \\
=|\xi| & +\int_{t \wedge \zeta_{\tau}}^{\zeta_{\tau}} \operatorname{sgn}\left(Y_{s}\right)\left(\bar{f}_{c}\left(s, Y_{s}\right)-f(s, 0)\right) d s+\int_{t \wedge \zeta_{\tau}}^{\zeta_{\tau}} \operatorname{sgn}\left(Y_{s}\right) f(s, 0) d s \\
& +\int_{t \wedge \zeta_{\tau}}^{\zeta_{\tau}} \operatorname{sgn}\left(Y_{s}\right)\left\{\left(\bar{g}_{c}\left(s, Y_{s}\right)-g(s, 0)\right) d A_{s}^{\mu}+\bar{g}_{c}(s, 0) d A_{s}\right\}-\int_{t \wedge \zeta_{\tau}}^{\zeta_{\tau}} \operatorname{sgn}\left(Y_{s-}\right) d M_{s} .
\end{aligned}
$$

From this, Eq. 3.15 and (P3), (P5) we get

$$
\left|Y_{t}\right|=E_{z}\left(\left|Y_{t}\right| \mid \mathcal{F}_{t}\right) \leq E_{z}\left(|\xi|+\int_{0}^{\zeta_{\tau}}\left(|f(s, 0)| d s+\left|\bar{g}_{c}(s, 0)\right| d A_{s}\right) \mid \mathcal{F}_{t}\right) \leq c
$$

which shows that in fact $(Y, M)$ is a solution of Eq. 3.15 with $\bar{f}_{c}$ replaced by $f$ and $\bar{g}_{c}$ replaced by $g$ 
Step 2. For $n \geq 0$, we set $\xi^{n}=T_{n}(\xi), f_{n}(t, y)=f(t, y)-f(t, 0)+T_{n}(f(t, 0))$ (with $\xi$, $f(t, y)$ defined in Step 1) and $A_{t}^{n}=\int_{0}^{t} \mathbf{1}_{\left\{A_{r}^{\mu} \leq n\right\}} d A_{r}^{\mu}$. By Step 1, for each $n \geq 0$ there exists a unique solution $\left(Y^{n}, M^{n}\right)$ of the BSDE

$$
\begin{aligned}
Y_{t}^{n}=\xi^{n} & +\int_{t \wedge \zeta_{\tau}}^{\zeta_{\tau}} f_{n}\left(s, Y_{s}^{n}\right) d s+\int_{t \wedge \zeta_{\tau}}^{\zeta_{\tau}}\left(g\left(s, Y_{s}^{n}\right)-g(s, 0)\right) d A_{s}^{\mu} \\
& +\int_{t \wedge \zeta_{\tau}}^{\zeta_{\tau}} T_{n} g(s, 0) d A_{s}^{n}-\int_{t \wedge \zeta_{\tau}}^{\zeta_{\tau}} d M_{s}^{n}, \quad t \geq 0
\end{aligned}
$$

on the space $\left(\Omega, \mathcal{F}, P_{z}\right)$ (as in Step 1, for brevity, in our notation we drop the dependence of $\left(Y^{n}, M^{n}\right)$ on $z$ ). For $m \geq n \geq 0$, we write $\delta Y=Y^{m}-Y^{n}, \delta M=M^{m}-M^{n}, \delta \xi=\xi^{m}-\xi^{n}$. Since $\mu \in \mathcal{R}^{+}\left(E_{0, T}\right), A^{m}$ is an increasing process. Therefore using the Meyer-Tanaka formula we obtain

$$
\begin{aligned}
\left|\delta Y_{t}\right| \leq|\delta \xi| & +\int_{t \wedge \zeta_{\tau}}^{\zeta_{\tau}} \operatorname{sgn}\left(\delta Y_{s}\right)\left(f_{m}\left(s, Y_{s}^{m}\right)-f_{n}\left(s, Y_{s}^{n}\right)\right) d s \\
& +\int_{t \wedge \zeta_{\tau}}^{\zeta_{\tau}} \operatorname{sgn}\left(\delta Y_{s}\right)\left(g\left(s, Y_{s}^{m}\right)-g\left(s, Y_{s}^{n}\right)\right) d A_{s}^{\mu} \\
& +\int_{t \wedge \zeta_{\tau}}^{\zeta_{\tau}} \operatorname{sgn}\left(\delta Y_{s}\right)\left\{T_{m} g(s, 0) d A_{s}^{m}-T_{n} g(s, 0) d A_{s}^{n}\right\}+\int_{t \wedge \zeta_{\tau}}^{\zeta_{\tau}} \operatorname{sgn}\left(\delta Y_{s-}\right) d(\delta M)_{s} .
\end{aligned}
$$

From the above and (P3), (P5) it follows that

$$
\begin{aligned}
\left|\delta Y_{t}\right| \leq|\delta \xi| & +\int_{t \wedge \zeta_{\tau}}^{\zeta_{\tau}}\left|T_{m} f(s, 0)-T_{n} f(s, 0)\right| d s+\int_{t \wedge \zeta_{\tau}}^{\zeta_{\tau}}\left|T_{m} g(s, 0)-T_{n} g(s, 0)\right| d A_{s}^{m} \\
& +\int_{t \wedge \zeta_{\tau}}^{\zeta_{\tau}}\left|T_{n} g(s, 0)\right| d\left(A_{s}^{m}-A_{s}^{n}\right)+\int_{t \wedge \zeta_{\tau}}^{\zeta_{\tau}} \operatorname{sgn}\left(\delta Y_{s-}\right) d(\delta M)_{s} .
\end{aligned}
$$

Hence

$$
\left|\delta Y_{t}\right|=E_{z}\left(\left|\delta Y_{t}\right| \mid \mathcal{F}_{t}\right) \leq E_{z}\left(\Psi^{n} \mid \mathcal{F}_{t}\right), \quad t \geq 0
$$

where

$$
\begin{aligned}
\Psi^{n}=|\xi| \mathbf{1}_{\{|\xi|>n\}}+\int_{0}^{\zeta_{\tau}}|f(t, 0)| \mathbf{1}_{\{|f(t, 0)|>n\}} d t & \\
& +\int_{0}^{\zeta_{\tau}}|g(t, 0)| \mathbf{1}_{\{|g(t, 0)|>n\}} d A_{t}^{\mu}+\int_{0}^{\zeta_{\tau}}|g(t, 0)| d\left(A_{t}^{m}-A_{t}^{n}\right) .
\end{aligned}
$$

Observe that from our assumptions on the data $\varphi, f, g, \mu$ it follows that $E_{z} \Psi^{n} \rightarrow 0$ as $n \rightarrow \infty$ for q.e. $z \in E_{0, T}$. By Eq. 3.18, $\|\delta Y\|_{z, 1} \leq E_{z} \Psi^{n}$, while by [4, Lemma 6.1], $E_{z} \sup _{t \leq T}\left|\delta Y_{t}\right|^{q} \leq(1-q)^{-1}\left(E_{z} \Psi^{n}\right)^{q}$ for every $q \in(0,1)$. Since the spaces $\mathcal{D}^{q}\left(P_{z}\right)$ and $\mathcal{L}^{1}\left(P_{z}\right)$ are complete, for q.e. $z \in E_{0, T}$ there exists a process $Y^{z}$ such that $Y^{z} \in \mathcal{D}^{q}\left(P_{z}\right)$ for $q \in(0,1), Y^{z}$ is of class D under $P_{z}$ and

$$
\left\|Y^{n}-Y^{z}\right\|_{1, z} \rightarrow 0, \quad E_{z} \sup _{0 \leq t \leq \zeta_{\tau}}\left|Y_{t}^{n}-Y_{t}^{z}\right|^{q} \rightarrow 0 .
$$

We have

$$
\begin{aligned}
\int_{0}^{\zeta_{\tau}}\left|f_{n}\left(t, Y_{t}^{n}\right)-f\left(t, Y_{t}^{z}\right)\right| d t \leq & \int_{0}^{\zeta_{\tau}}\left|f\left(t, Y_{t}^{n}\right)-f\left(t, Y_{t}^{z}\right)\right| d t \\
& +\int_{0}^{\zeta_{\tau}}|f(t, 0)| \mathbf{1}_{\{|f(t, 0)|>n\}} d t .
\end{aligned}
$$


Applying the Meyer-Tanaka formula we get (see the proof of Eq. 3.16)

$$
\left|Y_{t}^{n}\right| \leq E_{z}\left(|\xi|+\int_{0}^{\zeta_{\tau}}|f(s, 0)| d s+\int_{0}^{\zeta_{\tau}}|g(s, 0)| d A_{s}^{\mu} \mid \mathcal{F}_{t}\right)=: R_{t}, \quad t \geq 0 .
$$

For $k, N \in \mathbb{N}$, we set

$$
\begin{aligned}
\tau_{k, N}=\inf \{t \geq 0 & : R_{t} \geq k, \int_{0}^{t}(|f(s,-k)|+|f(s, k)|) d s \\
& \left.+\int_{0}^{t}(|g(s,-k)|+|g(s, k)|) d A_{s}^{\mu} \geq N\right\} \wedge \zeta_{\tau} .
\end{aligned}
$$

By Eq. 3.17,

$$
\begin{aligned}
Y_{t \wedge \tau_{k, N}}^{n}=E_{z}\left(Y_{\tau_{k, N}}^{n}+\int_{t \wedge \tau_{k, N}}^{\tau_{k, N}} f_{n}\left(s, Y_{s}^{n}\right) d s\right. \\
\left.\left.\quad+\int_{t \wedge \tau_{k, N}}^{\tau_{k, N}}\left\{g\left(s, Y_{s}^{n}\right)-g(s, 0)\right) d A_{s}^{\mu}+T_{n} g(s, 0) d A_{s}^{n}\right\} \mid \mathcal{F}_{t}\right) .
\end{aligned}
$$

From the definition of $\tau_{k, N}$ it follows that

$$
\int_{0}^{\tau_{k, N}}\left|f\left(t, Y_{t}^{n}\right)\right| d t+\int_{0}^{\tau_{k, N}}\left|g\left(t, Y_{t}^{n}\right)\right| d A_{t}^{\mu} \leq N .
$$

From this, (P2) and Eq. 3.19 one can deduce that

$$
\lim _{n \rightarrow \infty} E_{z} \int_{0}^{\tau_{k, N}}\left(\left|f_{n}\left(t, Y_{t}^{n}\right)-f\left(t, Y_{t}^{z}\right)\right| d t=0\right.
$$

and

$$
\lim _{n \rightarrow \infty} E_{z} \int_{0}^{\tau_{k, N}}\left\{\left|g\left(t, Y_{t}^{n}\right)-g(t, 0)\right| d A_{t}^{\mu}+\left|T_{n} g(t, 0)\right| d A_{t}^{n}\right\}=0 .
$$

By Doob's inequality (see, e.g., [21, Theorem 1.9.1]) and Eq. 3.19, for every $\varepsilon>0$ we have

$$
\lim _{n \rightarrow \infty} P_{x}\left(\sup _{t \leq T}\left|E_{z}\left(Y_{\tau_{k, N}}^{n}-Y_{\tau_{k, N}}^{z} \mid \mathcal{F}_{t}\right)\right|>\varepsilon\right) \leq \varepsilon^{-1} \lim _{n \rightarrow \infty} E_{z}\left|Y_{\tau_{k, N}}^{n}-Y_{\tau_{k, N}}^{z}\right|=0 .
$$

Similarly, by Eqs. 3.21, 3.22 and Doob's inequality,

$$
\lim _{n \rightarrow \infty} P_{z}\left(\sup _{t \leq T}\left|E_{z}\left(\int_{t \wedge \tau_{k, N}}^{\tau_{k, N}}\left(f\left(s, Y_{s}^{n}\right)-f\left(s, Y_{s}^{z}\right)\right) d s \mid \mathcal{F}_{t}\right)\right|>\varepsilon\right)=0
$$

and

$$
\lim _{n \rightarrow \infty} P_{z}\left(\sup _{t \leq T}\left|E_{z}\left(\int_{t \wedge \tau_{k, N}}^{\tau_{k, N}}\left(g\left(s, Y_{s}^{n}\right)-g\left(s, Y_{s}^{z}\right)\right) d A_{s}^{\mu} \mid \mathcal{F}_{t}\right)\right|>\varepsilon\right)=0
$$

for every $\varepsilon>0$. Letting $n \rightarrow \infty$ in Eq. 3.20 and using Eqs. 3.23-3.25 we conclude that

$$
Y_{t \wedge \tau_{k, N}}^{z}=E_{z}\left(Y_{\tau_{k, N}}^{z}+\int_{t \wedge \tau_{k, N}}^{\tau_{k, N}}\left\{f\left(s, Y_{s}^{z}\right) d s+g\left(s, Y_{s}^{z}\right) d A_{s}^{\mu}\right\} \mid \mathcal{F}_{t}\right) .
$$


We have

$$
\begin{aligned}
\int_{0}^{\zeta_{\tau}} \mid & f_{n}\left(t, Y_{t}^{n}\right)\left|d t+\int_{0}^{\zeta_{\tau}}\right| g\left(t, Y_{t}^{n}\right) \mid d A_{t}^{\mu} \\
\leq & \int_{0}^{\zeta_{\tau}}\left|f_{n}\left(t, Y_{t}^{n}\right)-f_{n}(t, 0)\right| d t+\int_{0}^{\zeta_{\tau}}\left|f_{n}(t, 0)\right| d t \\
& +\int_{0}^{\zeta_{\tau}}\left|g\left(t, Y_{t}^{n}\right)-g(t, 0)\right| d A_{t}^{\mu}+\int_{0}^{\zeta_{\tau}}|g(t, 0)| d A_{t}^{\mu} \\
= & -\int_{0}^{\zeta_{\tau}} \operatorname{sgn}\left(Y_{t}^{n}\right)\left(f_{n}\left(t, Y_{t}^{n}\right)-f_{n}(t, 0)\right) d t+\int_{0}^{\zeta_{\tau}}\left|f_{n}(t, 0)\right| d t \\
& -\int_{0}^{\zeta_{\tau}} \operatorname{sgn}\left(Y_{t}^{n}\right)\left(g\left(t, Y_{t}^{n}\right)-g(t, 0)\right) d A_{t}^{\mu}+\int_{0}^{\zeta_{\tau}}|g(t, 0)| d A_{t}^{\mu} .
\end{aligned}
$$

By the Meyer-Tanaka formula and Eq. 3.17,

$$
\begin{aligned}
\left|\xi^{n}\right|-\left|Y_{0}^{n}\right| \geq- & \int_{0}^{\zeta_{\tau}} \operatorname{sgn}\left(Y_{t}^{n}\right) f_{n}\left(t, Y_{t}^{n}\right) d t-\int_{0}^{\zeta_{\tau}} \operatorname{sgn}\left(Y_{t}^{n}\right) g\left(t, Y_{t}^{n}\right) d A_{t}^{\mu} \\
& -\int_{0}^{\zeta_{\tau}} \operatorname{sgn}\left(Y_{t}^{n}\right) T_{n} g(t, 0) d A_{t}^{n}-\int_{0}^{\zeta_{\tau}} \operatorname{sgn}\left(Y_{t-}^{n}\right) d M_{t} .
\end{aligned}
$$

Hence

$$
\begin{aligned}
& E_{z} \int_{0}^{\zeta_{\tau}}\left\{\left|f_{n}\left(t, Y_{t}^{n}\right)\right| d t+\left|g\left(t, Y_{t}^{n}\right)\right| d A_{t}^{\mu}\right\} \\
& \quad \leq E_{z}\left(\left|\xi^{n}\right|+2 \int_{0}^{\zeta_{\tau}}|f(t, 0)| d t+3 \int_{0}^{\zeta_{\tau}}|g(t, 0)| d A_{t}^{\mu}\right),
\end{aligned}
$$

so applying Fatou's lemma and Eq. 3.19 gives

$$
\begin{gathered}
E_{z} \int_{0}^{\zeta_{\tau}}\left\{\left|f\left(t, Y_{t}^{z}\right)\right| d t+\left|g\left(t, Y_{t}^{z}\right)\right| d A_{t}^{\mu}\right\} \\
\leq E_{z}\left(\left|\varphi\left(\mathbf{X}_{\zeta_{\tau}}\right)\right|+2 \int_{0}^{\zeta_{\tau}}|f(t, 0)| d t+3 \int_{0}^{\zeta_{\tau}}|g(t, 0)| d A_{t}^{\mu}\right)<\infty .
\end{gathered}
$$

Since $f(\cdot,-k), f(\cdot, k) \in q L^{1}\left(E_{0, T} ; m_{1}\right)$ and $g(\cdot,-k), g(\cdot, k) \in q L^{1}\left(E_{0, T} ; \mu\right), \tau_{k, N} \rightarrow \tau_{k}$ as $N \rightarrow \infty$, where

$$
\tau_{k}=\inf \left\{t \geq 0: R_{t} \geq k\right\} \wedge \zeta_{\tau} .
$$

Hence $Y_{\tau_{k, N}}^{z} \rightarrow Y_{\tau_{k}}^{z} P_{z}$-a.s., and consequently,

$$
\lim _{N \rightarrow \infty} E_{z}\left|Y_{\tau_{k, N}}^{z}-Y_{\tau_{k}}^{z}\right|=0
$$

since $Y^{z}$ is of class D. Letting $N \rightarrow \infty$ in Eq. 3.26 and using Eqs. 3.27, 3.28 and Doob's inequality we obtain

$$
Y_{t \wedge \tau_{k}}^{z}=E_{z}\left(Y_{\tau_{k}}^{z}+\int_{t \wedge \tau_{k}}^{\tau_{k}}\left\{f\left(s, Y_{s}^{z}\right) d s+g\left(s, Y_{s}^{z}\right) d A_{s}^{\mu}\right\} \mid \mathcal{F}_{t}\right) .
$$

Since $\tau_{k} \rightarrow \zeta_{\tau}$ as $k \rightarrow \infty$, letting $k \rightarrow \infty$ in Eq. 3.29 and repeating arguments used to prove (3.29) we get

$$
Y_{t}^{z}=E_{z}\left(\xi+\int_{t \wedge \zeta_{\tau}}^{\zeta_{\tau}}\left\{f\left(s, Y_{s}^{z}\right) d s+g\left(s, Y_{s}^{z}\right) d A_{s}^{\mu}\right\} \mid \mathcal{F}_{t}\right) .
$$


We may now repeat the reasoning following [18, (3.6)] with the process $V$ from [18] replaced by $\int_{0}^{*} g\left(t, Y_{t}^{z}\right) d A_{t}^{\mu}$ (see also the reasoning following Eq. 4.26 in the present paper) to prove that the pair $\left(Y^{z}, \tilde{M}^{z}\right)$, where $\tilde{M}^{z}$ is a càdlàg version of the martingale

$$
t \mapsto E_{z}\left(\varphi\left(\mathbf{X}_{\zeta_{\tau}}\right)+\int_{0}^{\zeta_{\tau}}\left\{f\left(\mathbf{X}_{s}, Y_{s}^{z}\right) d s+g\left(\mathbf{X}_{s}, Y_{s}^{z}\right) d A_{s}^{\mu}\right\} \mid \mathcal{F}_{t}\right)-\bar{u}\left(\mathbf{X}_{0}\right),
$$

is a solution of the BSDE

$$
Y_{t}^{z}=\varphi\left(\mathbf{X}_{\zeta_{\tau}}\right)+\int_{t \wedge \zeta_{\tau}}^{\zeta_{\tau}}\left\{f\left(\mathbf{X}_{s}, Y_{s}^{z}\right) d s+g\left(\mathbf{X}_{s}, Y_{s}^{z}\right) d A_{s}^{\mu}\right\}-\int_{t \wedge \zeta_{\tau}}^{\zeta_{\tau}} d \tilde{M}_{s}^{z}, \quad t \geq 0,
$$

on $\left(\Omega, \mathcal{F}, P_{z}\right)$. Furthermore, by [15, Remark 3.6], there exists a pair of processes $(Y, M)$ such that $\left(Y_{t}, M_{t}\right)=\left(Y^{z}, \tilde{M}_{t}^{z}\right), t \in\left[0, \zeta_{\tau}\right], P_{z}$-a.s. for q.e. $z \in E_{0, T}$. Let $u(z)=E_{z} Y_{0}$. Then the argument from the beginning of the proof of [14, Theorem 5.8] shows that $Y_{t}=u\left(\mathbf{X}_{t}\right)$, $t \in\left[0, \zeta_{\tau}\right]$, which implies that $M$ is a version of the martingale $M^{z}$ and that $(u(\mathbf{X}), M)$ is a solution of Eq. 3.30 for q.e. $z \in E_{0, T}$. In view of our convention made at the beginning of Section 2.2, this means that $(u(\mathbf{X}), M)$ is a solution of Eq. 3.4 on the space $\left(\Omega, \mathcal{F}, P_{z}\right)$ for q.e. $z \in E_{0, T}$. Of course, $u(\mathbf{X}) \in \mathcal{D}^{q}\left(P_{z}\right)$. Furthermore, $M$ is a uniformly integrable martingale under $P_{z}$, because under $P_{z}$ it is a version of the closed martingale $M^{z}$. Finally, since we know that $Y_{t}^{z}=u(\mathbf{X}), t \in\left[0, \zeta_{\tau}\right], P_{z}$-a.s., Eq. 3.13 follows immediately from Eq. 3.27. This completes the proof of part (ii) of the theorem.

Part (i) follows from (ii). Indeed, since $\mu \in \mathcal{R}\left(E_{0, T}\right)$ and we know that Eq. 3.27 is satisfied with $Y^{z}$ replaced by $u(\mathbf{X})$ and $M$ is a martingale under $P_{z}$ for q.e. $z \in E_{0, T}$, putting $t=0$ in Eq. 3.4 and then taking the expectation shows that $\bar{u}$ is a solution of Eq. 3.2. To show that $\bar{u}$ is unique one can argue as in the proof of [14, Theorem 5.8].

Remark 3.3 If $g$ does not depend on the last variable $y$, then in Theorem 3.2 we may replace the assumptions $\mu \in \mathcal{R}^{+}\left(E_{0, T}\right), g(\cdot, \cdot, 0) \cdot \mu \in \mathcal{R}\left(E_{0, T}\right)$ by the assumption $g \cdot \mu \in \mathcal{R}\left(E_{0, T}\right)$ (see [14, Theorem 5.8]).

Remark 3.4 (i) By [14, Proposition 3.4], the solution $u$ of Theorem 3.2 is quasi-continuous. (ii) Let the assumptions of Theorem 3.2 hold, and moreover, $f(\cdot, \cdot, 0) \in L^{1}\left(E_{0, T} ; m_{1}\right)$, $g(\cdot, \cdot, 0) \cdot \mu \in \mathcal{M}_{0, b}\left(E_{0, T}\right)$ and for some $\gamma \geq \alpha_{0}$ the form $\mathcal{E}_{\gamma}^{0, T}$ has the dual Markov property (for the definition of $\mathcal{E}^{0, T}$ see [14, Section 3.3]). Then by [14, Proposition 3.13] and Eq. 3.13,

$$
\begin{gathered}
\left\|f_{u}\right\|_{L^{1}\left(E_{0, T} ; m_{1}\right)}+\left\|g_{u} \cdot \mu\right\|_{T V} \leq c\left(\|\varphi\|_{L^{1}(E ; m)}+\|f(\cdot, \cdot, 0)\|_{L^{1}\left(E_{0, T} ; m_{1}\right)}\right. \\
\left.+\|g(\cdot, \cdot, 0) \cdot \mu\|_{T V}\right),
\end{gathered}
$$

where $\|\cdot\|_{T V}$ denotes the total variation norm. Therefore, by [14, Theorem 3.12], $u \in$ $L^{1}\left(E_{0, T} ; m_{1}\right), T_{k} u \in L^{2}(0, T ; V)$ for $k>0\left(T_{k} u\right.$ is defined by Eq. 3.14) and for every $k>0$ there is $C>0$ depending only on $k, \alpha, T$ such that

$$
\int_{0}^{T} B^{(t)}\left(T_{k} \bar{u}(t), T_{k} \bar{u}(t)\right) d t \leq C\left(\|\varphi\|_{L^{1}(E ; m)}+\|f(\cdot, 0)\|_{L^{1}\left(E_{0, T} ; m_{1}\right)}+\|g(\cdot, \cdot, 0) \cdot \mu\|_{T V}\right) .
$$

Moreover, if the forms $\left(B^{(t)}, V\right)$ are (non-symmetric) Dirichlet forms, then by [17, Theorem 4.5], $u$ is a renormalized solution of Eq. 3.2 in the sense defined in [17].

Remark 3.5 In Theorem 3.2 we have assumed that the AF $A^{\mu}$ is continuous. In the general case where $\mu \in \mathcal{R}^{+}\left(E_{0, T}\right)$ and $A^{\mu}$ is possibly discontinuous, one can prove the existence of a solution of Eq. 3.2 in the following sense: there exists $u: E_{T} \rightarrow \mathbb{R}$ such that $f_{u} \cdot m, g_{u} \cdot \mu \in$ 
$\mathcal{R}\left(E_{0, T}\right)$ and Eq. 3.5 is satisfied with $g_{u}$ replaced by $g_{\hat{u}}$, where $\hat{u}$ is the precise version of $u$ (for the notion of a precise version of a parabolic potential see [32]). In the paper we decided to provide the proof of less general result, because it suffices for the purposes of Sections 4 6 in which our main results are proved, and on the other hand, the proof of the general result is more technical than the proof of Theorem 3.2. Also note that by [14, Proposition 3.4], the solution $u$ described above is quasi-càdlàg.

\section{Convergence of BSDEs and Elliptic PDEs}

In this section, we assume that Eq. 2.9. We denote by $L$ the operator associated via (3.1) with the form $(B, V)$. We also assume that $\mu \in \mathcal{R}^{+}\left(E_{0, T}\right)$ does not depend on time and $f, g: E \times \mathbb{R} \rightarrow \mathbb{R}$, i.e. $f, g$ also do not depend on time. For $v \in E \rightarrow \mathbb{R}$, we set

$$
f_{v}(x):=f(x, v(x)), \quad g_{v}(x):=g(x, v(x)), \quad x \in E .
$$

To shorten notation, in what follows we denote $P_{0, x}$ by $P_{x}, E_{0, x}$ by $E_{x}$ and $\|\cdot\|_{(0, x) ; 1}$ by $\|\cdot\|_{x, 1}$. Under the measure $P_{x}$,

$$
\mathbf{X}_{t}=\left(t, X_{t}\right), \quad t \geq 0, \quad \zeta_{\tau}=T \wedge \zeta
$$

and

$$
A_{t}^{\mu}=A_{t}^{0, \tilde{\mu}}, \quad t \geq 0,
$$

where $\tilde{\mu}$ is determined by Eq. 2.6.

In the rest of the paper we say that some property is satisfied quasi-everywhere (q.e. for brevity) if the set of those $x \in E$ for which it does not hold is exceptional with respect to the form $(B, V)$.

Let $v \in S(E)$. We will say that a Borel measurable $F: E \rightarrow \mathbb{R}$ is $v$-quasi-integrable $\left(F \in q L^{1}(E ; v)\right.$ in notation) if for every $T>0, P_{x}\left(\int_{0}^{\zeta \wedge T}\left|F\left(X_{t}\right)\right| d A_{t}^{0, v}<\infty\right)=1$ for q.e. $x \in E$.

Note that in case $v=m$ the notion of quasi-integrability was introduced in [13, Section 2]. For a comparison of the notion of $m$-integrability and the notion of quasi-integrability in the analytic sense see [13, Remark 2.3].

In this section and Section 5, we assume that the data satisfy the following conditions.

(E1) $\varphi \in L^{1}(E ; m), \tilde{\mu} \in \mathcal{R}^{+}(E)$.

(E2) $f(\cdot, y), g(\cdot, y)$ are measurable for every $y \in \mathbb{R}$ and $f(x, \cdot), g(x, \cdot)$ are continuous for every $x \in E$.

(E3) $\quad\left(f(x, y)-f\left(x, y^{\prime}\right)\right)\left(y-y^{\prime}\right) \leq 0$ for all $y, y^{\prime} \in \mathbb{R}$ and $x \in E$.

(E4) $f(\cdot, 0) \cdot m \in \mathcal{R}(E)$ and $f(\cdot, y) \in q L^{1}(E ; m)$ for every $y \in \mathbb{R}$.

(E5) $\quad\left(g(x, y)-g\left(x, y^{\prime}\right)\right)\left(y-y^{\prime}\right) \leq 0$ for all $y, y^{\prime} \in \mathbb{R}$ and $x \in E$.

(E6) $g(\cdot, 0) \cdot \tilde{\mu} \in \mathcal{R}(E)$ and $g(\cdot, y) \in q L^{1}(E ; \tilde{\mu})$ for every $y \in \mathbb{R}$.

Definition Let $x \in E$. We say that a pair $\left(Y^{x}, M^{x}\right)$ is a solution of the BSDE

$$
Y_{t}^{x}=\int_{t \wedge \zeta}^{\zeta} f\left(X_{s}, Y_{s}^{x}\right) d s+\int_{t \wedge \zeta}^{\zeta} g\left(X_{s}, Y_{s}^{x}\right) d A_{s}^{\mu}-\int_{t \wedge \zeta}^{\zeta} d M_{s}^{x}, \quad t \geq 0,
$$

on the space $\left(\Omega, \mathcal{F}, P_{x}\right)$ if

(a) $\quad Y^{x}$ is an $\left(\mathcal{F}_{t}\right)$-progressively measurable process of class D under $P_{x}, Y_{t \wedge \zeta}^{x} \rightarrow 0, P_{x^{-}}$ a.s. as $t \rightarrow \infty$ and $M^{x}$ is an $\left(\mathcal{F}_{t}\right)$-local martingale under $P_{x}$ such that $M_{0}^{x}=0$, 
(b) For every $T>0, \int_{0}^{T}\left|f\left(X_{t}, Y_{t}^{x}\right)\right| d t<\infty, \int_{0}^{T}\left|g\left(X_{t}, Y_{t}^{x}\right)\right| d A_{t}^{\mu}<\infty, P_{x}$-a.s., and

$$
Y_{t}^{x}=Y_{T \wedge \zeta}^{x}+\int_{t \wedge \zeta}^{T \wedge \zeta} f\left(X_{s}, Y_{s}^{x}\right) d s+\int_{t \wedge \zeta}^{T \wedge \zeta} g\left(X_{s}, Y_{s}^{x}\right) d A_{s}^{\mu}-\int_{t \wedge \zeta}^{T \wedge \zeta} d M_{s}^{x}, \quad t \in[0, T] .
$$

Definition We say that $v: E \rightarrow \mathbb{R}$ is a solution of problem (1.2) with $\lambda=0$ if $f_{v} \cdot m \in$ $\mathcal{R}(E), g_{v} \cdot \tilde{\mu} \in \mathcal{R}(E)$ and for q.e. $x \in E$,

$$
v(x)=E_{x}\left(\int_{0}^{\zeta} f_{v}\left(X_{t}\right) d t+\int_{0}^{\zeta} g_{v}\left(X_{t}\right) d A_{t}^{\mu}\right) .
$$

Suppose that for some $x \in E$ for every $n>0$ there exists a solution $\left(Y^{n}, M^{n}\right)$ of the BSDE

$$
\begin{aligned}
Y_{t}^{n}=\mathbf{1}_{\{\zeta>n\}} \varphi\left(X_{n}\right)+\int_{t \wedge \zeta}^{n \wedge \zeta} f\left(X_{s}, Y_{s}^{n}\right) d s & \\
& +\int_{t \wedge \zeta}^{n \wedge \zeta} g\left(X_{s}, Y_{s}^{n}\right) d A_{s}^{\mu}-\int_{t \wedge \zeta}^{n \wedge \zeta} d M_{s}^{n}, \quad t \in[0, n], \quad P_{x} \text {-a.s. }
\end{aligned}
$$

on the probability space $\left(\Omega, \mathcal{F}, P_{x}\right)$. The solutions may depend on $x$ but for brevity, in our notation we drop the dependence of $Y^{n}, M^{n}$ on $x$. In what follows by $\tilde{Y}^{n}, \tilde{M}^{n}$ we denote the processes defined as

$$
\tilde{Y}_{t}^{n}=Y_{t}^{n}, \quad \tilde{M}_{t}^{n}=M_{t}^{n}, \quad t<n, \quad \tilde{Y}_{t}^{n}=0, \quad \tilde{M}_{t}^{n}=M_{n}^{n}, \quad t \geq n .
$$

Proposition 4.1 Assume that (E1)-(E6) are satisfied. For $0<n<m$, we set $\delta Y=\tilde{Y}^{m}-$ $\tilde{Y}^{n}$. Then for every $x \in E$,

$$
\begin{aligned}
\|\delta Y\|_{x, 1} \leq E_{x}\left(\mathbf{1}_{\{\zeta>m\}}\left|\varphi\left(X_{m}\right)\right|+\mathbf{1}_{\{\zeta>n\}}\left|\varphi\left(X_{n}\right)\right|\right. \\
\left.\quad+\int_{n \wedge \zeta}^{m \wedge \zeta}\left|f\left(X_{t}, 0\right)\right| d t+\int_{n \wedge \zeta}^{m \wedge \zeta}\left|g\left(X_{t}, 0\right)\right| d A_{t}^{\mu}\right)
\end{aligned}
$$

and

$$
\begin{aligned}
E_{x} \sup _{t \geq 0}\left|\delta Y_{t}\right|^{q} \leq \frac{1}{1-q} & \left(E_{x}\left(\mathbf{1}_{\{\zeta>m\}}\left|\varphi\left(X_{m}\right)\right|+\mathbf{1}_{\{\zeta>n\}} \mid \varphi\left(X_{n}\right)\right) \mid\right) \\
& \left.+E_{x} \int_{n \wedge \zeta}^{m \wedge \zeta}\left|f\left(X_{t}, 0\right)\right| d t+E_{x} \int_{n \wedge \zeta}^{m \wedge \zeta}\left|g\left(X_{t}, 0\right)\right| d A_{t}^{\mu}\right)^{q}
\end{aligned}
$$

for every $q \in(0,1)$. Moreover, for every $t \geq 0$,

$$
\begin{aligned}
& E_{x} \int_{0}^{t \wedge \zeta}\left|f\left(X_{s}, Y_{s}^{n}\right)\right| d s+E_{x} \int_{0}^{t \wedge \zeta}\left|g\left(X_{s}, Y_{s}^{n}\right)\right| d A_{s}^{\mu} \\
& \quad \leq E_{x}\left(\left|Y_{t}^{n}\right|+2 \int_{0}^{t \wedge \zeta}\left|f\left(X_{s}, 0\right)\right| d s+2 \int_{0}^{t \wedge \zeta}\left|g\left(X_{s}, 0\right)\right| d A_{s}^{\mu}\right) .
\end{aligned}
$$


Proof By Eq. 4.4,

$$
\begin{aligned}
Y_{t}^{n}= & Y_{0}^{n}-\int_{0}^{t \wedge \zeta}\left\{f\left(X_{s}, Y_{s}^{n}\right) d s+g\left(X_{s}, Y_{s}^{n}\right) d A_{s}^{\mu}\right\}+\int_{0}^{t \wedge \zeta} d M_{s}^{n} \\
= & Y_{0}^{n}-\int_{0}^{t}\left(\mathbf{1}_{[0, n \wedge \zeta]}(s) f\left(X_{s}, Y_{s}^{n}\right) d s+\mathbf{1}_{[0, n \wedge \zeta]}(s) g\left(X_{s}, Y_{s}^{n}\right) d A_{s}^{\mu}\right) \\
& +\int_{0}^{t} \mathbf{1}_{[0, n \wedge \zeta]}(s) d M_{s}^{n}, \quad t \in[0, n], P_{x} \text {-a.s. }
\end{aligned}
$$

From the above and the fact that the process $A^{\mu}$ is continuous it follows that the pair $\left(\tilde{Y}^{n}, \tilde{M}^{n}\right)$ defined by Eq. 4.5 satisfies

$$
\begin{aligned}
\tilde{Y}_{t}^{n}=Y_{0}^{n} & -\int_{0}^{t}\left(\mathbf{1}_{[0, n \wedge \zeta]}(s) f\left(X_{s}, \tilde{Y_{s}^{n}}\right) d s+\mathbf{1}_{[0, n \wedge \zeta]}(s) g\left(X_{s}, \tilde{Y_{s}^{n}}\right) d A_{s}^{\mu}\right) \\
& +\int_{0}^{t} d V_{s}^{n}+\int_{0}^{t} \mathbf{1}_{[0, n \wedge \zeta]}(s) d \tilde{M}_{s}^{n}, \quad t \geq 0,
\end{aligned}
$$

where

$$
V_{t}^{n}=0 \text { if } t<n, \quad V_{t}^{n}=-Y_{n}^{n} \text { if } t \geq n .
$$

Let $\delta \tilde{Y}=\tilde{Y}^{m}-\tilde{Y}^{n}$. By Eq. 4.10,

$$
\delta \tilde{Y}_{t}=\delta \tilde{Y}_{0}+K_{t}+\int_{0}^{t}\left(\mathbf{1}_{[0, m \wedge \zeta]}(s) d \tilde{M}_{s}^{m}-\mathbf{1}_{[0, n \wedge \zeta]}(s) d \tilde{M}_{s}^{n}\right), \quad t \geq 0, P_{x} \text {-a.s. }
$$

where

$$
\begin{aligned}
K_{t}=- & \int_{0}^{t} \mathbf{1}_{[0, n \wedge \zeta]}(s)\left(f\left(X_{s}, \tilde{Y_{s}^{m}}\right)-f\left(X_{s}, \tilde{Y_{s}^{n}}\right)\right) d s-\int_{0}^{t} \mathbf{1}_{(n \wedge \zeta, m \wedge \zeta]}(s) f\left(X_{s}, \tilde{Y_{s}^{m}}\right) d s \\
& -\int_{0}^{t} \mathbf{1}_{[0, n \wedge \zeta]}(s)\left(g\left(X_{s}, \tilde{Y_{s}^{m}}\right)-g\left(X_{s}, \tilde{Y_{s}^{n}}\right)\right) d A_{s}^{\mu} \\
& -\int_{0}^{t} \mathbf{1}_{(n \wedge \zeta, m \wedge \zeta]}(s) g\left(X_{s}, \tilde{Y_{s}^{m}}\right) d A_{s}^{\mu}+\int_{0}^{t} d\left(V_{s}^{m}-V_{s}^{n}\right) .
\end{aligned}
$$

By the Meyer-Tanaka formula (see [34, p. 216]), for $t<m$ we have

$$
\left|\delta \tilde{Y}_{m}\right|-\left|\delta \tilde{Y}_{t}\right| \geq \int_{t}^{m} \operatorname{sgn}\left(\delta \tilde{Y}_{s-}\right) d(\delta \tilde{Y})_{s},
$$

where $\operatorname{sgn}(x)=1$ if $x>0$ and $\operatorname{sgn}(x)=-1$ if $x \leq 0$. Therefore, for $t<m$,

$$
\left|\delta \tilde{Y}_{t}\right|=E_{x}\left(\left|\delta \tilde{Y}_{t}\right| \mid \mathcal{F}_{t}\right) \leq E_{x}\left(\left|\delta \tilde{Y}_{m}\right|-\int_{t}^{m} \operatorname{sgn}\left(\delta \tilde{Y}_{s-}\right) d K_{s} \mid \mathcal{F}_{t}\right) .
$$

From this it follows that for $t \in[0, m]$,

$$
\begin{aligned}
\left|\delta \tilde{Y}_{t}\right| \leq E_{x}\left(\left|\delta \tilde{Y}_{m}\right|\right. & +\int_{t}^{m} \mathbf{1}_{[0, n \wedge \zeta]}(s) \operatorname{sgn}\left(\delta \tilde{Y}_{s}\right)\left(f\left(X_{s}, \tilde{Y_{s}^{m}}\right)-f\left(X_{s}, \tilde{Y_{s}^{n}}\right)\right) d s \\
& +\int_{t}^{m} \mathbf{1}_{[0, n \wedge \zeta]}(s) \operatorname{sgn}\left(\delta \tilde{Y}_{s}\right)\left(g\left(X_{s}, \tilde{Y_{s}^{m}}\right)-g\left(X_{s}, \tilde{Y_{s}^{n}}\right)\right) d A_{s}^{\mu} \\
& +\int_{t}^{m} \mathbf{1}_{(n \wedge \zeta, m \wedge \zeta]}(s) \operatorname{sgn}\left(\delta Y_{s}\right) f\left(X_{s}, \tilde{Y_{s}^{m}}\right) d s \\
& \left.+\int_{t}^{m} \mathbf{1}_{(n \wedge \zeta, m \wedge \zeta]}(s) \operatorname{sgn}\left(\delta \tilde{Y}_{s}\right) g\left(X_{s}, \tilde{Y_{s}^{m}}\right) d A_{s}^{\mu}+\left|V_{m}^{m}\right|+\left|V_{n}^{n}\right| \mid \mathcal{F}_{t}\right) .
\end{aligned}
$$


By (E3),

$$
\int_{t}^{m} \mathbf{1}_{[0, n \wedge \zeta]}(s) \operatorname{sgn}\left(\delta \tilde{Y}_{s}\right)\left(f\left(X_{s}, \tilde{Y_{s}^{m}}\right)-f\left(X_{s}, \tilde{Y_{s}^{n}}\right)\right) d s \leq 0,
$$

whereas by (E5) and the fact that $A^{\mu}$ is increasing,

$$
\int_{t}^{m} \mathbf{1}_{[0, n \wedge \zeta]}(s) \operatorname{sgn}\left(\delta \tilde{Y}_{s}\right)\left(g\left(X_{s}, \tilde{Y_{s}^{m}}\right)-g\left(X_{s}, \tilde{Y}_{s}^{n}\right)\right) d A_{s}^{\mu} \leq 0 .
$$

Furthermore, since $\tilde{Y}_{t}^{n}=0$ for $t \geq n$, it follows from (E3) that

$$
\begin{aligned}
\int_{t}^{m} \mathbf{1}_{(n \wedge \zeta, m \wedge \zeta]}(s) \operatorname{sgn}\left(\delta \tilde{Y}_{s}\right) f\left(X_{s}, \tilde{Y_{s}^{m}}\right) d s \leq & \int_{t}^{m} \mathbf{1}_{(n \wedge \zeta, m \wedge \zeta]}(s) \operatorname{sgn}\left(\delta \tilde{Y}_{s}\right) f\left(X_{s}, 0\right) d s \\
& \leq \int_{n \wedge \zeta}^{m \wedge \zeta}\left|f\left(X_{s}, 0\right)\right| d s .
\end{aligned}
$$

Similarly, by (E5),

$$
\int_{t}^{m} \mathbf{1}_{(n \wedge \zeta, m \wedge \zeta]}(s) \operatorname{sgn}\left(\delta \tilde{Y}_{s}\right) g\left(X_{s}, \tilde{Y_{s}^{m}}\right) d A_{s}^{\mu} \leq \int_{n \wedge \zeta}^{m \wedge \zeta}\left|g\left(X_{s}, 0\right)\right| d A_{s}^{\mu} .
$$

Furthermore, $\delta \tilde{Y}_{m}=0$ and

$$
\left|V_{m}^{m}\right|+\left|V_{n}^{n}\right|=\left|Y_{m}^{m}\right|+\left|Y_{n}^{n}\right|=\mathbf{1}_{\{\zeta>m\}}\left|\varphi\left(X_{m}\right)\right|+\mathbf{1}_{\{\zeta>n\}}\left|\varphi\left(X_{n}\right)\right| .
$$

Therefore, for $t \in[0, m]$ we have

$$
\begin{aligned}
\left|\delta \tilde{Y}_{t}\right| \leq E_{x}\left(\mathbf{1}_{\{\zeta>m\}}\left|\varphi\left(X_{m}\right)\right|+\mathbf{1}_{\{\zeta>n\}}\left|\varphi\left(X_{n}\right)\right|\right. & \\
& \left.+\int_{n \wedge \zeta}^{m \wedge \zeta}\left|f\left(X_{s}, 0\right)\right| d s+\int_{n \wedge \zeta}^{m \wedge \zeta}\left|g\left(X_{s}, 0\right)\right| d A_{s}^{\mu} \mid \mathcal{F}_{t}\right)=: N_{t}
\end{aligned}
$$

This implies (4.6). By [4, Lemma 6.1],

$$
E_{x} \sup _{0 \leq t \leq m}\left|\delta \tilde{Y}_{t}\right|^{q} \leq(1-q)^{-1}\left(E_{x} N_{m}\right)^{q}
$$

which shows Eq. 4.7. Finally, to prove (4.8), we first observe that by the Meyer-Tanaka formula,

$$
E_{x}\left|Y_{t}^{n}\right|-E_{x}\left|Y_{0}^{n}\right| \geq E_{x} \int_{0}^{t} \operatorname{sgn}\left(Y_{s-}^{n}\right) d Y_{s}^{n} .
$$

By the above inequality and Eq. 4.9, for $t<n$ we have

$$
\begin{aligned}
& E_{x}\left|Y_{t}^{n}\right|-E_{x}\left|Y_{0}^{n}\right| \\
& \quad \geq-E_{x} \int_{0}^{t} \mathbf{1}_{\{[0, n \wedge \zeta]}(s) \operatorname{sgn}\left(Y_{s}^{n}\right)\left\{f\left(X_{s}, Y_{s}^{n}\right) d s+g\left(X_{s}, Y_{s}^{n}\right) d A_{s}^{\mu}\right\} .
\end{aligned}
$$

On the other hand, for every $t \geq 0$,

$$
\begin{aligned}
\int_{0}^{t}\left|g\left(X_{s}, Y_{s}^{n}\right)\right| d A_{s}^{\mu} & \leq \int_{0}^{t}\left|g\left(X_{s}, Y_{s}^{n}\right)-g\left(X_{s}, 0\right)\right| d A_{s}^{\mu}+\int_{0}^{t}\left|g\left(X_{s}, 0\right)\right| d A_{s}^{\mu} \\
& =-\int_{0}^{t} \operatorname{sgn}\left(Y_{s}^{n}\right)\left(g\left(X_{s}, Y_{s}^{n}\right)-g\left(X_{s}, 0\right)\right) d A_{s}^{\mu}+\int_{0}^{t}\left|g\left(X_{s}, 0\right)\right| d A_{s}^{\mu} \\
& \leq-\int_{0}^{t} \operatorname{sgn}\left(Y_{s}^{n}\right) g\left(X_{s}, Y_{s}^{n}\right) d A_{s}^{\mu}+2 \int_{0}^{t}\left|g\left(X_{s}, 0\right)\right| d A_{s}^{\mu}
\end{aligned}
$$


and similarly,

$$
\int_{0}^{t}\left|f\left(X_{s}, Y_{s}^{n}\right)\right| d s \leq-\int_{0}^{t} \operatorname{sgn}\left(Y_{s}^{n}\right) f\left(X_{s}, Y_{s}^{n}\right) d s+2 \int_{0}^{t}\left|f\left(X_{s}, 0\right)\right| d s,
$$

which when combined with Eq. 4.12 proves (4.8).

Proposition 4.2 Assume that (E1)-(E6) are satisfied and

$$
\lim _{t \rightarrow \infty} E_{x} \mathbf{1}_{\{\zeta>t\}}\left|\varphi\left(X_{t}\right)\right|=0 .
$$

Assume also for some $x \in E$ for each $n \in \mathbb{N}$ there exists a solution $\left(Y^{n}, M^{n}\right)$ of Eq. 4.4 on the space $\left(\Omega, \mathcal{F}, P_{x}\right)$. If

$$
E_{x} \int_{0}^{\zeta}\left|f\left(X_{t}, 0\right)\right| d t+E_{x} \int_{0}^{\zeta}\left|g\left(X_{t}, 0\right)\right| d A_{t}^{\mu}<\infty
$$

then there exists a solution $\left(Y^{x}, M^{x}\right)$ of Eq. 4.2 on $\left(\Omega, \mathcal{F}, P_{x}\right)$. Moreover, $Y^{x} \in \mathcal{D}^{q}\left(P_{x}\right)$ for $q \in(0,1), M^{x}$ is a uniformly integrable $\left(\mathcal{F}_{t}\right)$-martingale under $P_{x}$ and

$$
\begin{aligned}
& E_{x} \int_{0}^{\zeta}\left|f\left(X_{t}, Y_{t}^{x}\right)\right| d t+E_{x} \int_{0}^{\zeta}\left|g\left(X_{t}, Y_{t}^{x}\right)\right| d A_{t}^{\mu} \\
& \quad \leq 2 E_{x}\left(\int_{0}^{\zeta}\left|f\left(X_{t}, 0\right)\right| d t+\int_{0}^{\zeta}\left|g\left(X_{t}, 0\right)\right| d A_{t}^{\mu}\right) .
\end{aligned}
$$

Finally,

$$
\lim _{n \rightarrow \infty}\left\|Y^{n}-Y^{x}\right\|_{x, 1}=0
$$

and for every $q \in(0,1)$,

$$
\lim _{n \rightarrow \infty} E_{x} \sup _{t \geq 0}\left|Y_{t}^{n}-Y_{t}^{x}\right|^{q}=0
$$

Proof From Eqs. 4.6 and 4.13, 4.14 it follows that for every $x \in E,\left\|Y^{n}-Y^{m}\right\|_{x, 1} \rightarrow 0$ as $n, m \rightarrow \infty$. Hence there exists a process $Y \in \mathcal{L}^{1}\left(P_{x}\right)$ of class $\mathrm{D}$ such that Eq. 4.16 is satisfied. By Eqs. 4.7, 4.13 and 4.14, $\lim _{n, m \rightarrow \infty} E_{x} \sup _{t \geq 0}\left|Y_{t}^{n}-Y_{t}^{m}\right|^{q} \rightarrow 0$. Since the space $\mathcal{D}^{q}\left(P_{x}\right)$ is complete, the last convergence and Eq. 4.16 imply that $Y^{x} \in \mathcal{D}^{q}\left(P_{x}\right)$ and Eq. 4.17 is satisfied. From Eqs. 4.8, 4.16, 4.17 and Fatou's lemma it follows that for every $T>0$,

$$
\begin{aligned}
& E_{x} \int_{0}^{T \wedge \zeta}\left|f\left(X_{t}, Y_{t}^{x}\right)\right| d t+E_{x} \int_{0}^{T \wedge \zeta}\left|g\left(X_{t}, Y_{t}^{x}\right)\right| d A_{t}^{\mu} \\
\leq & 2 E_{x}\left(\left|Y_{T \wedge \zeta}^{x}\right|+\int_{0}^{T \wedge \zeta}\left|f\left(X_{t}, 0\right)\right| d s+\int_{0}^{T \wedge \zeta}\left|g\left(X_{t}, 0\right)\right| d A_{t}^{\mu}\right) .
\end{aligned}
$$

Since $\mathbf{1}_{\{n \geq \zeta\}} Y_{\zeta}^{n}=0 P_{x}$-a.s. for $n \in \mathbb{N}$, from Eq. 4.17 we conclude that $Y_{T \wedge \zeta}^{x} \rightarrow 0$ in probability $P_{x}$ as $T \rightarrow \infty$. As a consequence, since $Y^{x}$ is of class $\mathrm{D}, E_{x}\left|Y_{T \wedge \zeta}^{x}\right| \rightarrow 0$. Therefore letting $T \rightarrow \infty$ in the last inequality we get (4.15). Using Eq. 4.17 one can show that $\int_{0}^{\zeta}\left|g\left(X_{t}, Y_{t}^{n}\right)-g\left(X_{t}, Y_{t}^{x}\right)\right| d A_{t}^{\mu} \rightarrow 0$ in probability $P_{x}$ (see the proof of [12, (6.16)]). Set $F_{R}(t, x)=|f(t, x,-R)| \vee|f(t, x, R)|, G_{R}(t, x)=|g(t, x,-R)| \vee|g(t, x, R)|$ and for $N, R>0$ and $n \in \mathbb{N}$ define the stoping times

$$
\tau_{n, R}=\inf \left\{t \geq 0:\left|Y_{t}^{n}\right|>R\right\}, \quad \tau_{R}=\inf _{n \geq R} \tau_{n, R}
$$

and

$$
\sigma_{N, R}=\inf \left\{t \geq 0: \int_{0}^{t}\left(F_{R}\left(X_{s}\right) d s+G_{R}\left(X_{s}\right) d A_{s}^{\mu}\right)>N\right\}, \quad \delta_{N, R}=\sigma_{N, R} \wedge \tau_{R} .
$$


By Eq. 4.4, for $T<n$ we have

$$
\begin{gathered}
Y_{t \wedge \zeta \wedge \delta_{N, R}}^{n}=Y_{T \wedge \zeta \wedge \delta_{N, R}^{n}}^{n}+\int_{t \wedge \zeta \wedge \delta_{N, R}}^{T \wedge \zeta \wedge \delta_{N, R}}\left\{f\left(X_{s}, Y_{s}^{n}\right) d s+g\left(X_{s}, Y_{s}^{n}\right) d A_{s}^{\mu}\right\} \\
-\int_{t \wedge \zeta \wedge \delta_{N, R}}^{T \wedge \zeta \wedge \delta_{N, R}} d M_{s}^{n}, \quad t \in[0, T], \quad P_{x} \text {-a.s. }
\end{gathered}
$$

Since $Y_{t}^{n}=Y_{t \wedge \zeta}^{n}$ and $\int_{t \wedge \zeta \wedge \delta_{N, R}}^{T \wedge \zeta \wedge \delta_{N, R}} d M_{s}^{n}=\int_{t}^{T} d M_{s \wedge \zeta \wedge \delta_{N, R}}^{n}$ and the martingale $M^{n}$ stopped at $\zeta \wedge \delta_{N, R}$ is still a martingale (see [34, Theorem I.18]), it follows that

$$
Y_{t \wedge \zeta \wedge \delta_{N, R}}^{n}=E_{x}\left(Y_{T \wedge \zeta \wedge \delta_{N, R}}^{n}+\int_{t \wedge \zeta \wedge \delta_{N, R}}^{T \wedge \zeta \wedge \delta_{N, R}}\left\{f\left(X_{s}, Y_{s}^{n}\right) d s+g\left(X_{s}, Y_{s}^{n}\right) d A_{s}^{\mu}\right\} \mid \mathcal{F}_{t}\right) .
$$

By Doob's inequality (see, e.g., [21, Theorem 1.9.1]) and Eq. 4.16, for every $\varepsilon>0$ we have

$$
\begin{gathered}
\lim _{n \rightarrow \infty} P_{x}\left(\sup _{t \leq T}\left|E_{x}\left(Y_{T \wedge \zeta \wedge \delta_{N, R}}^{n}-Y_{T \wedge \zeta \wedge \delta_{N, R}}^{x} \mid \mathcal{F}_{t}\right)\right|>\varepsilon\right) \\
\leq \varepsilon^{-1} \lim _{n \rightarrow \infty} E_{x}\left|Y_{T \wedge \zeta \wedge \delta_{N, R}}^{n}-Y_{T \wedge \zeta \wedge \delta_{N, R}}^{x}\right|=0 .
\end{gathered}
$$

From the definition of $\delta_{N, R}$ and (E2), Eq. 4.17 it follows that

$$
\lim _{n \rightarrow \infty} E_{x} \int_{0}^{T \wedge \zeta \wedge \delta_{N, R}}\left\{\left|f\left(X_{s}, Y_{s}^{n}\right)-f\left(X_{s}, Y_{s}^{x}\right)\right| d s+\left|g\left(X_{s}, Y_{s}^{n}\right)-g\left(X_{s}, Y_{s}^{x}\right)\right| d A_{s}^{\mu}\right\}=0 .
$$

Hence, by Doob's inequality (see, e.g., [21, Theorem 1.9.1]),

$$
\lim _{n \rightarrow \infty} P_{x}\left(\sup _{t \leq T}\left|E_{x}\left(\int_{t \wedge \zeta \wedge \delta_{N, R}}^{T \wedge \zeta \wedge \delta_{N, R}}\left(f\left(X_{s}, Y_{s}^{n}\right)-f\left(X_{s}, Y_{s}^{x}\right)\right) d s \mid \mathcal{F}_{t}\right)\right|>\varepsilon\right)=0
$$

and

$$
\lim _{n \rightarrow \infty} P_{x}\left(\sup _{t \leq T}\left|E_{x}\left(\int_{t \wedge \zeta \wedge \delta_{N, R}}^{T \wedge \zeta \wedge \delta_{N, R}}\left(g\left(X_{s}, Y_{s}^{n}\right)-g\left(X_{s}, Y_{s}^{x}\right)\right) d A_{s}^{\mu} \mid \mathcal{F}_{t}\right)\right|>\varepsilon\right)=0
$$

for every $\varepsilon>0$. Letting $n \rightarrow \infty$ in Eq. 4.18 and using Eqs. 4.17 and 4.19-4.21 we conclude that $P_{x}$-a.s.

$$
Y_{t \wedge \delta_{N, R}}^{x}=E_{x}\left(Y_{T \wedge \zeta \wedge \delta_{N, R}}^{x}+\int_{t \wedge \zeta \wedge \delta_{N, R}}^{T \wedge \zeta \wedge \delta_{N, R}}\left\{f\left(X_{s}, Y_{s}^{x}\right) d s+g\left(X_{s}, Y_{s}^{x}\right) d A_{s}^{\mu}\right\} \mid \mathcal{F}_{t}\right)
$$

for $t \in[0, T]$. By (E4), $F_{R} \in q L^{1}\left(E_{0, T} ; m_{1}\right)$, and by (E6), $G_{R} \in q L^{1}\left(E_{0, T} ; \mu\right)$. Therefore $\sigma_{N, R} \nearrow \tau_{R} P_{x}$-a.s. as $N \rightarrow \infty$ for each fixed $R>0$. Hence $Y_{T \wedge \zeta \wedge \delta_{N, R}}^{x} \rightarrow Y_{T \wedge \zeta \wedge \tau_{R}}^{x} P_{x^{-}}$ a.s. as $N \rightarrow \infty$, and consequently $E_{x}\left|Y_{T \wedge \zeta \wedge \delta_{N, R}}^{x}-Y_{T \wedge \zeta \wedge \tau_{R}}^{x}\right| \rightarrow 0$ since $Y^{x}$ is of class D. From the last convergence and Doob's inequality it follows that for every $\varepsilon>0$,

$$
\lim _{N \rightarrow \infty} P_{x}\left(\sup _{t \leq T}\left|E_{x}\left(Y_{T \wedge \zeta \wedge \delta_{N, R}}^{x}-Y_{T \wedge \zeta \wedge \tau_{R}}^{x} \mid \mathcal{F}_{t}\right)\right|>\varepsilon\right)=0 .
$$

Therefore letting $N \rightarrow \infty$ in Eq. 4.22 and using Eq. 4.15 we show that $P_{x}$-a.s.,

$$
Y_{t}^{x}=E_{x}\left(Y_{T \wedge \zeta \wedge \tau_{R}}^{x}+\int_{t \wedge \zeta \wedge \tau_{R}}^{T \wedge \zeta \wedge \tau_{R}}\left\{f\left(X_{s}, Y_{s}^{x}\right) d s+g\left(X_{s}, Y_{s}^{x}\right) d A_{s}^{\mu}\right\} \mid \mathcal{F}_{t}\right), \quad t \in[0, T] .
$$


We now show that $\tau_{R} \nearrow \infty P_{x}$-a.s. as $R \rightarrow \infty$. To see this, let us suppose that $P_{x}\left(\sup _{R>0} \tau_{R} \leq M\right)>\varepsilon$ for some $M, \varepsilon>0$. Then

$$
P_{x}\left(\forall R>0 \quad \sup _{n \geq R} \sup _{t \leq M}\left|Y_{t}^{n}\right| \geq R\right)>\varepsilon .
$$

Clearly,

$$
\begin{aligned}
P_{x}\left(\forall_{R>0} \sup _{n \geq R} \sup _{t \leq M}\left|Y_{t}^{n}\right| \geq R\right) \leq & P_{x}\left(\forall_{R>0} \sup _{n \geq R} \sup _{t \leq M}\left|Y_{t}^{n}-Y_{t}\right| \geq R / 2\right) \\
& +P_{x}\left(\forall_{R>0} \sup _{t \leq M}\left|Y_{t}\right| \geq R / 2\right) \\
= & P\left(\forall_{R>0} \sup _{n \geq R} \sup _{t \leq M}\left|Y_{t}^{n}-Y_{t}\right| \geq R / 2\right) .
\end{aligned}
$$

By Eq. 4.17, taking a subsequence if necessary, we may assume that $\sup _{t \leq M}\left|Y_{t}^{n}-Y_{t}\right| \rightarrow 0$ $P_{x}$-a.s. Therefore the random variable $Z=\sup _{n \geq 0} \sup _{t \leq M}\left|Y_{t}^{n}-Y_{t}\right|$ is finite a.s., which when combined with Eq. 4.25 contradicts (4.24). This proves that $\tau_{R} \nearrow \propto P_{x}$-a.s. Now, letting $R \rightarrow \infty$ and repeating argument used to prove (4.23), we get (4.23) with $T \wedge \zeta \wedge \tau_{R}$ replaced by $T \wedge \zeta$. Since we know that $E_{x}\left|Y_{T \wedge \zeta}^{x}\right| \rightarrow 0$ as $T \rightarrow \infty$, letting $T \rightarrow \infty$ in this equation (i.e. in Eq. 4.23 with $T \wedge \zeta$ ) and repeating once again the argument used to prove (4.23) we get

$$
Y_{t}^{x}=E_{x}\left(\int_{t \wedge \zeta}^{\zeta}\left\{f\left(X_{s}, Y_{s}^{x}\right) d s+g\left(X_{s}, Y_{s}^{x}\right) d A_{s}^{\mu}\right\} \mid \mathcal{F}_{t}\right), \quad t \geq 0, \quad P_{x} \text {-a.s. }
$$

Hence

$$
Y_{t}^{x}=\int_{t \wedge \zeta}^{\zeta} f\left(X_{s}, Y_{s}^{x}\right) d s+\int_{t \wedge \zeta}^{\zeta} g\left(X_{s}, Y_{s}^{x}\right) d A_{s}^{\mu}-\int_{t \wedge \zeta}^{\zeta} d M_{s}^{x}, \quad t \geq 0, \quad P_{x} \text {-a.s., }
$$

where $M^{x}$ is a càdlàg version of the martingale

$$
t \mapsto E_{x}\left(\int_{0}^{\zeta} f\left(X_{s}, Y_{s}^{x}\right) d s+\int_{0}^{\zeta} g\left(X_{s}, Y_{s}^{x}\right) d A_{s}^{\mu} \mid \mathcal{F}_{t}\right)-Y_{0}^{x} .
$$

Indeed, by Eq. 4.26,

$$
\begin{aligned}
Y_{t}^{x}=E_{x} & \left(\int_{0}^{\zeta} f\left(X_{s}, Y_{s}^{x}\right) d s+\int_{0}^{\zeta} g\left(X_{s}, Y_{s}^{x}\right) d A_{s}^{\mu} \mid \mathcal{F}_{t}\right) \\
& -\int_{0}^{t \wedge \zeta} f\left(X_{s}, Y_{s}^{x}\right) d s-\int_{0}^{t \wedge \zeta} g\left(X_{s}, Y_{s}^{x}\right) d A_{s}^{\mu}, \quad t \geq 0,
\end{aligned}
$$

that is

$$
Y_{t}^{x}=Y_{0}^{x}+M_{t}^{x}-\int_{0}^{t \wedge \zeta} f\left(X_{s}, Y_{s}^{x}\right) d s-\int_{0}^{t \wedge \zeta} g\left(X_{s}, Y_{s}^{x}\right) d A_{s}^{\mu}, \quad t \geq 0 .
$$

From the above it follows that $M_{t \wedge \zeta}^{x}=M_{t}^{x}, t \geq 0$, and moreover, that

$$
Y_{t}^{x}=Y_{T \wedge \zeta}+\int_{t \wedge \zeta}^{T \wedge \zeta} f\left(X_{s}, Y_{s}^{x}\right) d s+\int_{t \wedge \zeta}^{T \wedge \zeta} g\left(X_{s}, Y_{s}^{x}\right) d A_{s}^{\mu}-\int_{t \wedge \zeta}^{T \wedge \zeta} d M_{s}^{x}, \quad t \geq 0 .
$$

Letting $T \rightarrow \infty$ and using the fact that $Y_{T \wedge \zeta}^{x} \rightarrow Y_{\zeta}^{x}=0 P_{x}$-a.s. we obtain (4.27). Thus the pair $\left(Y^{x}, M^{x}\right)$ is a solution of Eq. 4.2. 
Theorem 4.3 Assume (2.9) and assume that $f, g, \mu$ do not depend on time and satisfy (E1)-(E6).

(i) There exists a unique solution $v$ of problem (1.2) with $\lambda=0$.

(ii) Let

$$
M_{t}^{x}=E_{x}\left(\int_{0}^{\zeta} f_{v}\left(X_{r}\right) d r+\int_{0}^{\zeta} g_{v}\left(X_{r}\right) d A_{r}^{\mu} \mid \mathcal{F}_{t}\right)-v\left(X_{0}\right), \quad t \geq 0 .
$$

Then there is a càdlàg $\left(\mathcal{F}_{t}\right)$-adapted process $M$ such that $M_{t}=M_{t}^{x}, t \geq 0, P_{x}$-a.s. for q.e $x \in E$ and for q.e. $x \in E$ the pair $(v(X), M)$ is a unique solution of Eq. 4.2 on the space $\left(\Omega, \mathcal{F}, P_{x}\right)$. Moreover, $v(X) \in \mathcal{D}^{q}\left(P_{x}\right)$ for $q \in(0,1)$ and $M$ is a uniformly integrable martingale under $P_{x}$ for q.e. $x \in E$.

Proof We first prove part (ii). The uniqueness of a solution of Eq. 4.2 follows easily from (E3), (E5) and the fact that $\mu$ is positive. To see this it suffices to modify slightly the proof of [15, Proposition 3.1]. To prove the existence of a solution, we first note that by Theorem 3.2, for q.e. $x \in E$ for every $n \in \mathbb{N}$ there exists a unique solution $\left(Y^{n}, M^{n}\right)$ of the BSDE (4.4) with $\varphi \equiv 0$ on the space $\left(\Omega, \mathcal{F}, P_{x}\right)$. Since $f(\cdot, 0) \cdot m, g(\cdot, 0) \cdot \tilde{\mu} \in \mathcal{R}(E)$, condition (4.14) is satisfied for q.e. $x \in E$. Therefore, by Proposition 4.2, for q.e. $x \in E$ there exist a solution $\left(Y^{x}, \tilde{M}^{x}\right)$ of BSDE (4.2). In fact, $Y^{x}$ is given by Eq. 4.26 and $\tilde{M}^{x}$ is a càdlàg version of the martingale (4.28). Repeating step by step the proof of [15, Theorem 4.7] one can show that there is a pair of càdlàg processes $(Y, M)$ not depending on $x$ such that $\left(Y_{t}, M_{t}\right)=\left(Y_{t}^{x}, \tilde{M}_{t}^{x}\right), t \geq 0, P_{x}$-a.s. for q.e. $x \in E$, and secondly, that in fact $Y=v(X)$, where $v(x)=E_{x} Y_{0}$. This shows that the pair $(v(X), M)$ is a solution of Eq. 4.2 on the space $\left(\Omega, \mathcal{F}, P_{x}\right)$ for q.e. $x \in E$. By Proposition $4.2, v(X) \in \mathcal{D}^{q}\left(P_{x}\right)$ for $q \in(0,1)$, and $M$ is a uniformly integrable $\left(\mathcal{F}_{t}\right)$-martingale under $P_{x}$. This completes the proof of (ii). Part (i) follows immediately from (ii), because $g_{v} \cdot \mu \in \mathcal{R}(E)$ and Eq. 4.15 is satisfied with $Y^{x}$ replaced by $v(X)$, so for q.e. $x \in E$ we can integrate with respect to $P_{x}$ both sides of Eq. 4.2 with $t=0$ and $Y^{x}$ replaced by $v(X)$.

Remark 4.4 If $g$ does not depend on the last variable $y$, then in Theorem 4.3 we may replace the assumptions $\tilde{\mu} \in \mathcal{R}^{+}(E), g(\cdot, 0) \cdot \tilde{\mu} \in \mathcal{R}(E)$ by the assumption $g \cdot \tilde{\mu} \in \mathcal{R}(E)$ (see $[18$, Theorem 3.8]).

Remark 4.5 (i) By [15, Lemma 4.3], the solution $v$ of Eq. 1.2 appearing in Theorem 4.3 is quasi-continuous.

(ii) In addition to the hypotheses of Theorem 4.3 let us assume that $(B, V)$ is a transient Dirichlet form and $f(\cdot, 0) \in L^{1}(E, m), g(\cdot, 0) \cdot \tilde{\mu} \in \mathcal{M}_{b}(E)$, where $\tilde{\mu}$ is determined by Eq. 2.6. Then by Eq. 4.15, Lemma 2.2, the fact that $Y_{t}^{x}=v\left(X_{t}\right), t \geq 0, P_{x}$-a.s. and [18, Lemma 2.6] (see also [15, Lemma 5.4]),

$$
\left\|f_{v}\right\|_{L^{1}(E ; m)}+\left\|g_{v} \cdot \tilde{\mu}\right\|_{T V} \leq\|f(\cdot, 0)\|_{L^{1}(E ; m)}+\|g(0, \cdot) \cdot \tilde{\mu}\|_{T V} .
$$

Therefore, by [18, Theorem 4.2] (see also [15, Proposition 5.9]), $f_{v} \in L^{1}(E ; m), T_{k} v$ belongs to the extended Dirichlet space $V_{e}$ and for every $k>0$,

$$
B\left(T_{k} v, T_{k} v\right) \leq k\left(\|f(\cdot, 0)\|_{L^{1}(E ; m)}+\|g(0, \cdot) \cdot \tilde{\mu}\|_{T V}\right) .
$$

Moreover, if $(B, V)$ is a (non-symmetric) Dirichlet form satisfying the strong sector condition, then by [17, Theorem 3.5], $v$ is a renormalized solution of problem (1.2) in the sense defined in [17]. 
Remark 4.6 If a family $\left\{B^{(t)}, t \in \mathbb{R}\right\}$ satisfies the assumptions of Section 2 , then for every $\lambda>0$ the family $\left\{B_{\lambda}^{(t)}, t \in \mathbb{R}\right\}$, where $B_{\lambda}^{(t)}(\varphi, \psi)=B^{(t)}(\varphi, \psi)+\lambda(\varphi, \psi)_{H}$, satisfies these assumptions as well. Therefore all the results of Sections 3 and 4 apply to the operators associated with $B_{\lambda}^{(t)}$ and to the Markov process associated with the form $\mathcal{E}_{\lambda}$ defined by Eqs. 2.4, 2.5 but with $B^{(t)}$ replaced by $B_{\lambda}^{(t)}$.

\section{Large Time Asymptotics}

In this section, as in Section 4, we assume that Eq. 2.9 is satisfied and the data $f, g, \mu$ do not depend on time. We denote by $L$ the operator corresponding to $(B, V)$. We continue to write $P_{x}$ for $P_{0, x}$ and $E_{x}$ for $E_{0, x}$, and as in Section 4, the abbreviation "q.e." means quasi-everywhere with respect to the capacity determined by $(B, V)$.

Suppose that for every $T>0$ there exists a unique solution $u_{T}$ of Eq. 3.2 with $L$ and the data $f, g, \mu$ satisfying the above assumptions. By Remark 3.1, by putting

$$
u(t, x)=\bar{u}_{T}(t, x)=u_{T}(T-t, x), \quad t \in[0, T], x \in E,
$$

we define a probabilistic solution $u$ of Eq. 1.1, i.e. solution of the problem

$$
\left\{\begin{array}{l}
\partial_{t} u-L u=f(x, u)+g(x, u) \cdot \mu \quad \text { in }(0, \infty) \times E, \\
u(0, \cdot)=\varphi \text { on } E .
\end{array}\right.
$$

Our goal is to prove that under suitable assumptions, $u(t, x) \rightarrow v(x)$ as $t \rightarrow \infty$ for q.e. $x \in E$, where $v$ is a solution of Eq. 1.2 with $\lambda=0$, i.e. solution of the problem

$$
-L v=f(x, v)+g(x, v) \cdot \tilde{\mu} \quad \text { in } E,
$$

where $\tilde{\mu}$ is determined by Eq. 2.6. We will also estimate the rate of the convergence. The proofs of these results rely on the results of Section 4 . The main idea is as follows. We have

$$
u(t, x)=u_{T}(T-t, x), \quad t \in[0, T], x \in E,
$$

where $u_{T}$ is a solution of the problem

$$
\partial_{t} u_{T}+L u_{T}=-f\left(x, u_{T}\right)-g\left(x, u_{T}\right), \quad u_{T}(T)=\varphi .
$$

In particular, putting $t=T$, we get $u(T, x)=u_{T}(0, x)$. Hence, by Eq. 3.5,

$$
u(T, x)=E_{x}\left(\varphi\left(\mathbf{X}_{T \wedge \zeta}\right)+\int_{0}^{T \wedge \zeta} f_{u_{T}}\left(\mathbf{X}_{t}\right) d t+\int_{0}^{T \wedge \zeta} g_{u_{T}}\left(\mathbf{X}_{t}\right) d A_{t}^{\mu}\right)
$$

because $\zeta_{\tau}=T \wedge \zeta$ under the measure $P_{x}$. On the other hand, by Lemma 2.2,

$$
v(x)=E_{x}\left(\int_{0}^{\zeta} f_{v}\left(X_{t}\right) d t+\int_{0}^{\zeta} g_{v}\left(X_{t}\right) d A_{t}^{0, \tilde{\mu}}\right) .
$$

Therefore our problem reduces to showing that the right-hand side of Eq. 5.5 converges to the right-hand side of Eq. 5.6 as $T \rightarrow \infty$, and to estimating the difference between the two expressions by some function of $T$.

In what follows, we denote by $\left(P_{t}\right)_{t \geq 0},\left(R_{\alpha}\right)_{\alpha>0}$ the semigroup and the resolvent associated with the process $\mathbb{M}^{(0)}=\left(X, P_{x}\right)$ with life time $\zeta^{0}=\zeta$ (see Section 2.2), i.e.

$$
P_{t} f(x)=E_{x} f\left(X_{t}\right), \quad R_{\alpha} f(x)=E_{x} \int_{0}^{\infty} e^{-\alpha t} f\left(X_{t}\right) d t, \quad x \in E, f \in \mathcal{B}_{b}(E) .
$$


For $v \in \mathcal{R}(E)$, we set

$$
R_{\alpha} v(x)=E_{x} \int_{0}^{\zeta} e^{-\alpha t} d A_{t}^{0, v}=E_{x} \int_{0}^{\infty} e^{-\alpha t} d A_{t}^{0, v},
$$

where $A^{0, v}$ is the continuous $\mathrm{AF}$ of $\mathbb{M}^{(0)}$ associated with $v$ in the Revuz sense. Note that if $(B, V)$ is transient, then $R_{\alpha} v$ is defined for $\alpha=0$.

Before stating our main result, let us note that with the convention made at the beginning of Section 2.2, $E_{x} \mathbf{1}_{\{\zeta>t\}} \psi\left(X_{t}\right)=P_{t} \psi(x)$ for Borel measurable $\psi \in L^{1}(E ; m), t \geq 0$. Therefore (4.13) is equivalent to

$$
\lim _{t \rightarrow \infty} P_{t}|\varphi|(x)=0 .
$$

Clearly, assumption (4.14) is equivalent to

$$
R_{0}|f(\cdot, 0)|(x)+R_{0}(|g(\cdot, 0)| \cdot \tilde{\mu})(x)<\infty .
$$

By remarks given in Section 2.2, if $f(\cdot, 0) \cdot m \in \mathcal{R}(E)$ and $g(\cdot, 0) \cdot \tilde{\mu} \in \mathcal{R}(E)$, then (5.8) is satisfied for q.e. $x \in E$.

Theorem 5.1 Assume that the assumptions of Theorem 4.3 hold, and moreover, Eq. 5.7 is satisfied. Let $u$ be a solution of Eq. 5.1 and $v$ be a solution of Eq. 5.2. Then

$$
\lim _{T \rightarrow \infty} u(T, x)=v(x)
$$

for q.e. $x \in E$. In fact, for q.e. $x \in E$,

$$
|u(T, x)-v(x)| \leq 3 P_{T}|\varphi|(x)+3 P_{T}\left(R_{0}(|f(\cdot, 0)|+|g(\cdot, 0)| \cdot \tilde{\mu})\right)(x)
$$

for all $T>0$.

Proof Let $Y^{T}$ be the first component of the solution of Eq. 4.4 (with $T=n$ ) and $Y$ be the first component of the solution of Eq. 4.2. Since Eq. 4.14 is satisfied for q.e. $x \in E$, applying Proposition 4.2 we conclude that for every $q \in(0,1)$,

$$
\lim _{T \rightarrow \infty} E_{x}\left|Y_{0}^{T}-Y_{0}\right|^{q}=0
$$

for q.e. $x \in E$. On the other hand, by Theorem 3.2 and Theorem 4.3, for q.e. $x \in E$ we have

$$
Y_{t}^{T}=u_{T}\left(\mathbf{X}_{t}\right), \quad Y_{t}=v\left(X_{t}\right), \quad t \geq 0, \quad P_{x} \text {-a.s., }
$$

where $u_{T}$ is a solution of Eq. 5.4 and $v$ is a solution of Eq. 4.3. In particular, for q.e. $x \in E$,

$$
Y_{0}^{T}=u_{T}(0, x), \quad Y_{0}=v(x), \quad P_{x} \text {-a.s. }
$$

But $u_{T}(0, x)=u(T, x)$ by Eq. 5.3. Hence

$$
|u(T, x)-v(x)|^{q}=\left|u_{T}(0, x)-v(x)\right|^{q}=E_{x}\left|Y_{0}^{T}-Y_{0}\right|^{q}
$$

for $T>0$. Therefore (5.11) implies (5.9). To show Eq. 5.10, we first observe that by Eqs. 5.11 and 5.12,

$$
|u(T, x)-v(x)|^{q}=\lim _{m \rightarrow \infty} E_{x}\left|Y_{0}^{T}-Y_{0}^{m}\right|^{q},
$$


whereas by Eqs. 4.7 and 4.13,

$$
\begin{gathered}
\lim _{m \rightarrow \infty} E_{x}\left|Y_{0}^{T}-Y_{0}^{m}\right|^{q} \leq \frac{1}{1-q}\left(E_{x} \mathbf{1}_{\{\zeta>T\}} \mid \varphi\left(X_{T}\right)\right)\left|+\int_{T \wedge \zeta}^{\zeta}\right| f\left(X_{t}, 0\right) \mid d t \\
\left.+\int_{T \wedge \zeta}^{\zeta}\left|g\left(X_{t}, 0\right)\right| d A_{t}^{\mu}\right)^{q} .
\end{gathered}
$$

By Lemma 2.2,

$$
E_{x} \int_{T \wedge \zeta}^{\zeta}\left|g\left(X_{t}, 0\right)\right| d A_{t}^{\mu}=E_{x} \int_{T}^{\infty}\left|g\left(X_{t}, 0\right)\right| d A_{t}^{0, \tilde{\mu}},
$$

so by the Markov property of $\mathbb{M}^{(0)}$,

$$
E_{x} \int_{T \wedge \zeta}^{\zeta}\left|g\left(X_{t}, 0\right)\right| d A_{t}^{\mu}=P_{T}\left(R_{0}(|g(\cdot, 0)| \cdot \tilde{\mu})\right)(x) .
$$

Similarly, since $\int_{0}^{t}\left|f\left(X_{s}, 0\right)\right| d s=A_{t}^{|f(\cdot, 0)| \cdot m}$ for $t \geq 0$, we have

$$
E_{x} \int_{T \wedge \zeta}^{\zeta}\left|f\left(X_{t}, 0\right)\right| d t=P_{T}\left(R_{0}|f(\cdot, 0)|\right)(x) .
$$

Combining (5.13)-(5.16) yields (5.10) but with constant 3 replaced by $(1-q)^{-1 / q}$ with arbitrary $q \in(0,1)$. This proves (5.10) since $(1-q)^{-1 / q} \rightarrow e$ as $q \downarrow 0$.

Let $\lambda \geq 0$ and let $L^{\lambda}$ denote the operator associated with the form $\left(B_{\lambda}, V\right)$, i.e.

$$
L^{\lambda}=L^{0}-\lambda
$$

where $L^{0}$ is the operator associated with $\left(B_{0}, V\right)=(B, V)$. Let $\left(P_{t}^{\lambda}\right),\left(R_{\alpha}^{\lambda}\right)$ denote the semigroup and the resolvent associated with the Hunt process corresponding to $\left(B_{\lambda}, V\right)$. It is well known that for $\psi \in L^{1}(E ; m), \mu \in \mathcal{R}(E)$ we have

$$
P_{t}^{\lambda} \psi(x)=e^{-\lambda t} P_{t}^{0} \psi(x), \quad R_{\alpha}^{\lambda} \mu(x)=R_{\alpha+\lambda}^{0} \mu(x)
$$

for q.e. $x \in E$. Therefore from Theorem 5.1 we immediately get the following corollary.

Corollary 5.2 Let the assumptions of Theorem 5.1 hold. Let $u, v$ be solutions of Eqs. 5.1 and 5.2, respectively, with $L=L^{\lambda}$ defined by Eq. 5.17. Then for q.e. $x \in E$,

$$
|u(T, x)-v(x)| \leq 3 e^{-\lambda T}\left(P_{T}^{0}|\varphi|(x)+P_{T}^{0}\left(R_{\lambda}^{0}(|f(\cdot, 0)|+|g(\cdot, 0)| \cdot \tilde{\mu})\right)(x)\right)
$$

for all $T>0$.

Remark 5.3 The results of Sections 3-5 can be carried over to quasi-regular forms. Indeed, if the forms $\{B(t), t \in[0, T]\}$ are quasi-regular, then by [35, Theorem IV.2.2], there exists a special standard process $\mathbf{M}$ properly associated in the resolvent sense with the time dependent form defined by Eq. 2.4. One can check that all the results of Sections 3 and 4 hold true for such a process. This is because in their proofs the fact that $\mathbf{M}$ is a Hunt process is not used and the results of [14] on which we rely in the proofs of Section 3 hold for quasiregular forms $\left(B^{(t)}, V\right)$ (see [14, Remark 4.4]). Similarly, the results of [18] on which we rely in Section 4 hold for quasi-regular form $(B, V)$. As a consequence, Theorem 5.1 holds true in the case of quasi regular form $(B, V)$ (its proof for such forms requires no changes). 


\section{Applications}

In this section, we give four quite different examples of forms $(B, V)$ and measures $\mu$ for which Theorem 5.1 applies.

\subsection{Classical local Dirichlet forms}

In this subsection, we assume that $E=D$, where $D$ is a nonempty connected bounded open subset of $\mathbb{R}^{d}$ with $d \geq 2$. We denote by $m$ the Lebesgue measure on $D$. We consider the classical form $(B, V)$ on $H=L^{2}(D ; m)$ defined as

$$
B(\varphi, \psi)=\frac{1}{2} \int_{D}(\nabla \varphi, \nabla \psi) d x, \quad \varphi, \psi \in V .
$$

We will consider two cases: $V=H_{0}^{1}(D)$ and $V=H^{1}(D)$.

Equations with Dirichlet boundary conditions Let $V=H_{0}^{1}(D)$. It is well known that $(B, V)$ is a regular Dirichlet form on $H$ (see [11, Example 1.2.3]). The operator $L$ associated with $(B, V)$ in the sense of Eq. 3.1 is $\frac{1}{2} \Delta$ with the Dirichlet boundary condition (see [11, Example 1.3.1]). The process $\mathbb{M}^{(0)}=\left(X, P_{x}\right)$ associated with $(B, V)$ in the resolvent sense is the Brownian motion killed upon leaving $D$ (see [11, Example 4.4.1]). Its life time is equal to $\tau_{D}=\inf \left\{t>0: X_{t} \notin D\right\}$.

We consider the problems

$$
\partial_{t} u-\frac{1}{2} \Delta u+h(u)|\nabla u|^{2}=\mu,\left.\quad u\right|_{(0, \infty) \times \partial D}=0, \quad u(0, \cdot)=\varphi
$$

and

$$
-\frac{1}{2} \Delta v+h(v)|\nabla v|^{2}=\tilde{\mu},\left.\quad u\right|_{\partial D}=0,
$$

where $\varphi \in L^{1}(D ; m)$ is nonnegative, $\mu=d t \otimes \tilde{\mu}$ with $\tilde{\mu} \in \mathcal{M}_{0, b}^{+}(D)$ and $h: \mathbb{R} \rightarrow \mathbb{R}$ is a continuous function satisfying the "sign condition", i.e.

$$
\forall s \in \mathbb{R}, \quad h(s) s \geq 0 .
$$

The model example is $h(s)=s, s \in \mathbb{R}$. In Eqs. 6.2 and 6.3 gradient of the solution appears, so they are more general than the equations studied in Sections 3-5. We shall see, however, that they are closely related to equations of the forms (5.1), (5.2).

We first give definitions of probabilistic solutions of Eqs. 6.2, 6.3.

Definition (a) We say that $u:(0, \infty) \times D \rightarrow \mathbb{R}$ is a probabilistic solution of Eq. 6.2 if for every $T>0$ the function $\bar{u}$ defined as $\bar{u}(t, x)=u(T-t, x),(t, x) \in D_{T}$, is a probabilistic solution of the problem

$$
\partial_{t} \bar{u}+\frac{1}{2} \Delta \bar{u}-h(\bar{u})|\nabla \bar{u}|^{2}=-\mu,\left.\quad \bar{u}\right|_{(0, T) \times \partial D}=0, \quad \bar{u}(T, \cdot)=\varphi,
$$

i.e. $h(\bar{u})|\nabla \bar{u}|^{2} \in \mathcal{R}\left(D_{0, T}\right)$ and for q.e. $z \in D_{0, T}$,

$$
\bar{u}(z)=E_{z}\left(\varphi\left(\mathbf{X}_{\zeta_{\tau}}\right)-\int_{0}^{\zeta_{\tau}} h(\bar{u})\left(\mathbf{X}_{t}\right)\left|\nabla \bar{u}\left(\mathbf{X}_{t}\right)\right|^{2} d t+\int_{0}^{\zeta_{\tau}} d A_{t}^{\mu}\right) .
$$


(b) We say that $v: D \rightarrow \mathbb{R}$ is a probabilistic solution of Eq. 6.3 if $h(v)|\nabla v|^{2} \in \mathcal{R}(E)$ and for q.e. $x \in D$,

$$
v(x)=E_{x}\left(-\int_{0}^{\zeta} h(v)\left(X_{t}\right)\left|\nabla v\left(X_{t}\right)\right|^{2} d t+\int_{0}^{\zeta} d A_{t}^{0, \tilde{\mu}}\right) .
$$

Let

$$
G(s)=2 \int_{0}^{s} h(t) d t, \quad \Phi(s)=\int_{0}^{s} \exp (-G(t)) d t, \quad s \in \mathbb{R},
$$

and let $H: \Phi(\mathbb{R}) \rightarrow \mathbb{R}$ be defined as

$$
H(s)=\exp \left(-G\left(\Phi^{-1}(s)\right)\right) .
$$

The function $\Phi$ is strictly increasing on $\mathbb{R}$, and by Eq. $6.4, G$ is nondecreasing on $[0, \infty)$. We set $\Phi(\infty)=\lim _{s \rightarrow \infty} \Phi(s), G(\infty)=\lim _{s \rightarrow \infty} G(s)$, and we define $\hat{H}: \mathbb{R} \rightarrow \mathbb{R}$ by

$$
\begin{cases}\hat{H}(s)=H(s), s \in[0, \infty), & \text { if } \Phi(\infty)=\infty, \\ \hat{H}(s)=H(s), s \in[0, \Phi(\infty)] \text { and } \hat{H}(s)=e^{-G(\infty)}, s>\Phi(\infty), & \text { if } \Phi(\infty)<\infty \\ \hat{H}(s)=H(0), & \text { if } s<0\end{cases}
$$

Notice that $\hat{H}$ is continuous and nonincreasing on $\mathbb{R}$, and $0 \leq \hat{H} \leq 1$. Therefore $g:=\hat{H}$ satisfies the hypotheses (E2), (E5) and (E6).

In Proposition 6.2 below we show that probabilistic solutions of problems (6.2), (6.3) are closely related to the probabilistic solutions of problems

$$
\partial_{t} w-\frac{1}{2} \Delta w=\hat{H}(w) \cdot \mu,\left.\quad w\right|_{(0, \infty) \times \partial D}=0, \quad w(0, \cdot)=\Phi(\varphi)
$$

and

$$
-\frac{1}{2} \Delta \tilde{w}=\hat{H}(\tilde{w}) \cdot \tilde{\mu},\left.\quad \tilde{w}\right|_{\partial D}=0
$$

We start with the observation that in fact, in the above equations, one can replace $\hat{H}$ by $H$.

Remark 6.1 If $w$ is a solution of Eq. 6.7, then $0 \leq w \leq \Phi(\infty)$ q.e. on $(0, \infty) \times D$. Thus, we can replace $\hat{H}$ by $H$ in Eq. 6.7. Similarly, if $\tilde{w}$ is a solution of Eq. 6.8, then $0 \leq \tilde{w} \leq \Phi(\infty)$ q.e. on $D$. Thus, we can replace $\hat{H}$ by $H$ in Eq. 6.8 .

We provide the proof for Eq. 6.7. The proof for Eq. 6.8 is similar. Let $T>0$ and $\bar{w}(t, x)=w(T-t, x)$. By [16, Proposition 3.7], for q.e. $z \in D_{0, T}$ the pair

$$
\left(Y_{t}, Z_{t}\right)=\left(\bar{w}\left(\mathbf{X}_{t}\right), \nabla \bar{w}\left(\mathbf{X}_{t}\right)\right), \quad t \in\left[0, \zeta_{\tau}\right],
$$

is a solution of the BSDE

$$
Y_{t}=\Phi\left(\varphi\left(\mathbf{X}_{\zeta_{\tau}}\right)\right)+\int_{t \wedge \zeta_{\tau}}^{\zeta_{\tau}} \hat{H}\left(Y_{s}\right) d A_{s}^{\mu}-\int_{t \wedge \zeta_{\tau}}^{\zeta_{\tau}} Z_{s} d W_{s}, \quad t \in\left[0, \zeta_{\tau}\right],
$$

under the measure $P_{z}$, where $W$ is some Wiener process starting from $z$ under $P_{z}$ (In different words, in the case where the form (6.1) is considered, if $w$ is a probabilistic solution of Eq. 6.7, then the martingale $M$ appearing in Theorem 3.2 (with the data from Eq. 6.7) has the representation $M_{t}=\int_{0}^{t} Z_{r} d W_{r}$ with $Z$ as above). Since, by assumption, $\varphi \geq 0$, we have $\Phi \circ \varphi \geq 0$, so from Eq. 6.9 it follows that $\bar{w} \geq 0$ q.e. on $D_{0, T}$. Since $T>0$ was arbitrary, $w \geq 0$ q.e. on $(0, \infty) \times D$. Since $w$ is quasi-continuous, it is finite q.e., so $w \leq \Phi(\infty)$ q.e. 
on $(0, \infty) \times D$ if $\Phi(\infty)=\infty$. Suppose now that $\Phi(\infty)<\infty$. To show that $w \leq \Phi(\infty)$, we first assume additionally that

$$
\int_{0}^{\infty} h(s) d s=\infty
$$

Choose $\left\{a_{n}\right\} \subset[0, \infty)$ such that $a_{n} \nearrow \Phi(\infty)$, By Eq. 6.9 and the Meyer-Tanaka formula, for q.e. $z \in E_{0, T}$ we have

$$
\begin{aligned}
\left(\bar{w}(z)-a_{n}\right)^{+} & \left.\leq E_{z}\left(\Phi \circ \varphi\left(X_{T}\right)-a_{n}\right)^{+}+\int_{0}^{\zeta_{\tau}} \mathbf{1}_{\left\{\bar{w}\left(\mathbf{X}_{s}\right)>a_{n}\right\}} \hat{H}\left(\bar{w}\left(\mathbf{X}_{s}\right)\right) d A_{s}^{\mu}\right) \\
& \left.\leq E_{z}\left(\Phi \circ \varphi\left(X_{T}\right)-a_{n}\right)^{+}+\int_{0}^{\zeta_{\tau}} H\left(a_{n}\right) d A_{s}^{\mu}\right) .
\end{aligned}
$$

By Eq. 6.10, $H\left(a_{n}\right) \searrow 0$, so letting $n \rightarrow \infty$ in Eq. 6.11 yields $(\bar{w}(z)-\Phi(\infty))^{+}=0$. Since $T>0$ was arbitrary, this implies that $w \leq \Phi(\infty)$ q.e. on $(0, \infty) \times D$. We now show how to dispense with the assumption (6.10). Let $h_{n}(x)=h(x)+(1 / n) \arctan x$ and $w_{n}$ be a solution of Eq. 6.7 with $\hat{H}$ replaced by $\hat{H}_{n}$ defined as $\hat{H}$ but with $h$ replaced by $h_{n}$. By what has already been proved $w_{n} \leq \Phi(\infty)$ q.e. on $(0, \infty) \times D$. Set $\bar{w}_{n}(t, x)=w_{n}(T-t, x)$. By using estimates of the form (3.13) we show that $\bar{w}_{n} \rightarrow \bar{w}$ q.e. on $D_{0, T}$. Consequently, $\bar{w} \leq \Phi(\infty)$ q.e. on $D_{0, T}$, so $w \leq \Phi(\infty)$ q.e. on $(0, \infty) \times D$.

Assertions (ii) and (iii) of Proposition 6.2 may be viewed as probabilistic reformulation of known analytic facts relating (6.2), (6.3) to (6.7), (6.8) (see, e.g., [20]) or [24, Remark 2.17]).

Proposition 6.2 Assume that $\varphi \in L^{1}(D ; m)$ is nonnegative, $\mu \in \mathcal{M}_{0, b}^{+}(D)$ and $h: D \rightarrow \mathbb{R}$ is a continuous function satisfying (6.4). Then

(i) There exists a unique solution u of problem (6.2) and a unique solution $v$ of problem (6.3). Moreover, $0 \leq u \leq \Phi(\infty) \geq 0$ q.e. on $(0, \infty) \times D$ and $0 \leq v \leq \Phi(\infty)$ q.e. on $D$.

(ii) $u$ is a probabilistic solution of Eq. 6.2 if and only if $w=\Phi(u)$ is a solution of Eq. 6.7 .

(iii) $v$ is a probabilistic solution of Eq. 6.3 if and only if $\tilde{w}=\Phi(v)$ is a solution of Eq. 6.8.

Proof We first prove (ii). Let $w$ be a solution of Eq. 6.7. For fixed $T>0$, we define $\bar{w}$ and $(Y, Z)$ as in Remark 6.1. We know that $0 \leq \bar{w} \leq \Phi(\infty)$ q.e. on $D_{0, T}$. Let $\bar{u}=\Phi^{-1}(\bar{w})$. Since $\Phi^{-1}$ is of class $C^{2}$, applying Itô's formula we get

$$
\begin{aligned}
\bar{u}\left(\mathbf{X}_{\zeta_{\tau}}\right)-\bar{u}\left(\mathbf{X}_{0}\right) & =\Phi^{-1}\left(Y_{\zeta_{\tau}}\right)-\Phi^{-1}\left(Y_{0}\right) \\
& =\int_{0}^{\zeta_{\tau}}\left(\Phi^{-1}\right)^{\prime}\left(Y_{t}\right) d Y_{t}+\frac{1}{2} \int_{0}^{\zeta_{\tau}}\left(\Phi^{-1}\right)^{\prime \prime}\left(Y_{t}\right) d\langle Y\rangle_{t} .
\end{aligned}
$$

But

$$
\left(\Phi^{-1}\right)^{\prime}=\frac{1}{\Phi^{\prime}\left(\Phi^{-1}\right)}, \quad\left(\Phi^{-1}\right)^{\prime \prime}=-\frac{1}{\left(\Phi^{\prime}\left(\Phi^{-1}\right)\right)^{2}} \cdot \Phi^{\prime \prime}\left(\Phi^{-1}\right) \cdot \frac{1}{\Phi^{\prime}\left(\Phi^{-1}\right)}
$$

a.e. with respect to the Lebesgue measure. Hence

$$
\begin{aligned}
\bar{u}\left(\mathbf{X}_{0}\right)=\bar{u} & \left(\mathbf{X}_{\zeta_{\tau}}\right)+\int_{0}^{\zeta_{\tau}} \frac{1}{\Phi^{\prime}(\bar{u})} H\left(\bar{w}\left(\mathbf{X}_{t}\right)\right) d A_{t}^{\mu} \\
& \quad-\int_{0}^{\zeta_{\tau}} \frac{1}{\Phi^{\prime}(\bar{u})} \nabla \bar{w}\left(\mathbf{X}_{t}\right) d W_{t}+\frac{1}{2} \int_{0}^{\zeta_{\tau}} \frac{\Phi^{\prime \prime}(\bar{u})}{\left(\Phi^{\prime}(\bar{u})\right)^{3}}|\nabla \bar{w}|^{2}\left(\mathbf{X}_{t}\right) d t .
\end{aligned}
$$


Since $\bar{u}\left(\mathbf{X}_{\zeta_{\tau}}\right)=\varphi\left(\mathbf{X}_{\zeta_{\tau}}\right)$ and

$$
\frac{\Phi^{\prime \prime}}{\left(\Phi^{\prime}\right)^{3}}=-\frac{2 h}{\left(\Phi^{\prime}\right)^{2}}, \quad \Phi^{\prime}(\bar{u})=H(\bar{w}), \quad \nabla \bar{w}=\Phi^{\prime}(\bar{u}) \nabla \bar{u},
$$

we have

$$
\bar{u}\left(\mathbf{X}_{0}\right)=\varphi\left(\mathbf{X}_{\zeta_{\tau}}\right)+\int_{0}^{\zeta_{\tau}} d A_{t}^{\mu}-\int_{0}^{\zeta_{\tau}} h(\bar{u})|\nabla \bar{u}|^{2}\left(\mathbf{X}_{t}\right) d t-\int_{0}^{\zeta_{\tau}} \nabla \bar{u}\left(\mathbf{X}_{t}\right) d W_{t} .
$$

Taking the expectation with respect to $P_{x}$ we see that $\bar{u}=\Phi^{-1}(\bar{w})$ is a probabilistic solution of Eq. 6.5. Hence $u=\Phi^{-1}(w)$ is a probabilistic solution of Eq. 6.2.

To prove the opposite implication, we first note that if $u$ is a solution of Eq. 6.2, then for every $T>0$, for q.e. $z \in D_{0, T}$ the pair

$$
\left(\tilde{Y}_{t}, \tilde{Z}_{t}\right)=\left(\bar{u}\left(\mathbf{X}_{t}\right), \nabla \bar{u}\left(\mathbf{X}_{t}\right)\right), \quad t \in\left[0, \zeta_{\tau}\right],
$$

is a solution of the BSDE

$$
\tilde{Y}_{t}=\varphi\left(\mathbf{X}_{\zeta_{\tau}}\right)-\int_{t \wedge \zeta_{\tau}}^{\zeta_{\tau}} h\left(\tilde{Y}_{s}\right)\left|\tilde{Z}_{s}\right|^{2} d s+\int_{t \wedge \zeta_{\tau}}^{\zeta_{\tau}} d A_{s}^{\mu}-\int_{t \wedge \zeta_{\tau}}^{\zeta_{\tau}} Z_{s} d W_{s}, \quad t \in\left[0, \zeta_{\tau}\right],
$$

under the measure $P_{z}$. In case $h(\bar{u})|\nabla \bar{u}|^{2} \in L^{1}\left(D_{0, T} ; m_{1}\right)$ this follows directly from [16, Proposition 3.7], while in case $h(\bar{u})|\nabla \bar{u}|^{2} \cdot m \in \mathcal{R}\left(D_{0, T}\right)$ follows from [16, Proposition 3.7] by simple approximation. Put $\bar{w}=\Phi(\bar{u})$. Applying Itô's formula we show that the pair

$$
\left(Y_{t}, Z_{t}\right)=\left(\bar{w}\left(\mathbf{X}_{t}\right), \nabla \bar{w}\left(\mathbf{X}_{t}\right)\right), \quad t \in\left[0, \zeta_{\tau}\right],
$$

is a solution of Eq. 6.9. From this it follows that $w$ is a solution of Eq. 6.7. This completes the proof of (ii).

The proof of (iii) is similar to that of (ii). We apply Itô's formula and the fact that in case of the form (6.1), the martingale $M$ appearing in Theorem 4.3 has the representation $M_{t}=\int_{0}^{t} Z_{s} d W_{s}, t \geq 0$, with $Z_{t}=\nabla v\left(X_{t}\right)$ if we consider Eq. 6.3, and with $Z_{t}=\nabla \tilde{w}\left(X_{t}\right)$ if we consider (6.8) (for the representation property for $M$ see [13, Theorem 3.5].

We now show (i). We know that $g:=\hat{H}$ satisfies the hypotheses (E2), (E5) and (E6). Therefore, by Theorem 3.2, there exists a unique solution $w$ of Eq. 6.7, while by Theorem 4.3, there exists a unique solution $\tilde{w}$ of Eq. 6.8. Therefore (i) follows from (ii), (iii) and Remark 6.1.

Remark 6.3 Assume that $\varphi \in L^{1}(D)$ is nonnegative, $\tilde{\mu}(d x)=\beta(x) d x$ for some $\beta \in$ $L^{1}(D)$ and $h$ is a continuous function satisfying (6.4). Moreover, assume that there exist $L, \delta>0$ such that $h(s) s \geq \delta$ for $s \in \mathbb{R}$ such that $|s| \geq L$.

(i) In [3] it is proved that under the above assumptions there exists a weak solution $v \in H_{0}^{1}(D)$ of Eq. 6.3 such that $h(v)|\nabla v|^{2} \in L^{1}(D ; m)$. A quasi-continuous version of $v$, which we still denote by $v$, is a probabilistic solution of Eq. 6.3. Indeed, since for every bounded $w \in H_{0}^{1}$ we have $B(v, w)=\int_{D}\left(h(v)|\nabla v|^{2}+\beta\right) w d x, v$ is a solution of problem (6.3) in the sense of duality (see [15, Section 5] for the definition). Therefore, by [15, Proposition 5.1], $v$ is a probabilistic solution of Eq. 6.3.

(ii) By the results proved in [33], there exists a weak solution $\bar{u} \in L^{2}\left(0, T ; H_{0}^{1}(D)\right)$ of problem (6.5) such that $h(\bar{u})|\nabla \bar{u}|^{2} \in L^{1}\left(D_{T} ; m_{1}\right)$. Its quasi-continuous version is a probabilistic solution of Eq. 6.5. This follows from the fact that it is a solution of Eq. 6.5 in the sense of duality (see [14, Section 4] for the definition), and hence, by [14, Corollary 4.2], a probabilistic solution of Eq. 6.5 . 
Proposition 6.4 Let $\varphi, h$ satisfy the assumptions of Proposition 6.2, and let $\mu(d x)=$ $\beta(x) m(d x)$ for some nonnegative $\beta \in L^{1}(D ; m)$. Then

(i) For q.e. $x \in D, u(t, x) \rightarrow v(x)$ as $t \rightarrow \infty$.

(ii) $u(t, \cdot) \rightarrow v$ in $L^{1}(D ; m)$ as $t \rightarrow \infty$.

Proof In the proof we adopt the notation from the proof of Proposition 6.2. We know that $w=\Phi(u)$ is nonnegative and solves (6.2) with $H$ replaced by $\hat{H}$. We also know that the initial condition $\Phi \circ \varphi$ and coefficients $f=0, g:=\hat{H}$ of that equation satisfy the assumptions (E1)-(E6). Moreover, we shall see in the proof of Proposition 6.6 (in a more general situation where $\Delta$ is replaced by the fractional Laplacian $\Delta^{\alpha / 2}$ ) that Eq. 5.7 with $\varphi$ replaced by $\Phi \circ \varphi$ is satisfied. Hence, by Theorem 5.1,w(t,x) $\rightarrow \tilde{w}(x)$ as $t \rightarrow \infty$ for q.e. $x \in D$. Therefore part (i) follows from Proposition 6.2 and the fact that $\Phi^{-1}$ is continuous. To prove part (ii), we first note that for every $T>0, \bar{w} \geq 0$ q.e. on $D_{0, T}$, so $\bar{u} \geq 0$ q.e. on $D_{0, T}$. Consequently, $h(\bar{u}) \geq 0$ q.e. on $D_{0, T}$ since $h$ satisfies (6.4). Therefore from Eq. 6.6 it follows that for q.e. $(s, x) \in D_{0, T}$,

$$
\bar{u}(s, x) \leq E_{s, x}\left(\varphi\left(\mathbf{X}_{\zeta_{\tau}}\right)+\int_{0}^{\zeta_{\tau}} d A_{t}^{\mu}\right)=: \overline{\hat{u}}(s, x) .
$$

The function $\hat{u}$ defined as $\hat{u}(t, x)=\overline{\hat{u}}(T-t, x),(t, x) \in D_{T}$, is a solution of Eq. 6.2 with $h \equiv 0$. By Theorem 5.1, $\hat{u}(t, x) \rightarrow \hat{v}(x)$ as $t \rightarrow \infty$ for q.e. $x \in D$, where $\hat{v}$ is a solution of Eq. 6.3 with $h \equiv 0$. In fact, by Eq. 5.10 (see the proof of Proposition 6.6 for details),

$$
|\hat{u}(t, x)-\hat{v}(x)| \leq C t^{-d / 2}\left(\|\varphi\|_{L^{1}(D ; m)}+\|\beta\|_{L^{1}(D ; m)}\right), \quad t>0,
$$

for q.e. $x \in D$. Since $D$ is bounded, it follows that $\hat{u}(t, \cdot) \rightarrow \hat{v}$ in $L^{1}(D ; m)$ as $t \rightarrow \infty$. From this and the fact that $0 \leq u(t, \cdot) \leq \hat{u}(t, \cdot)$ we conclude that the family $\{u(t, \cdot)\}$ is uniformly integrable, which together with (i) proves (ii).

By using a completely different method, part (ii) of the above proposition was proved in [20, Theorem 3.3] under the assumption that $h \in C^{1}(\mathbb{R})$ and $h^{\prime}(s)>0$ for $s \in \mathbb{R}$.

Equations with Neumann boundary conditions Let $D$ be a bounded Lipschitz domain in $\mathbb{R}^{d}, d \geq 3$. Set $E=\bar{D}$. Let $H=L^{2}(\bar{D} ; m)$, where $m$ is the Lebesgue measure on $\bar{D}$, and let $V=H^{1}(D)$. It is known that $(B, V)$ defined by Eq. 6.1 is a regular Dirichlet form on $H$ (see [11, Example 4.5.3]). The operator $L$ associated with $(B, V)$ in the sense of Eq. 3.1 is $\frac{1}{2} \Delta$ with the Neumann boundary condition, while the process $\mathbb{M}^{(0)}=\left(X, P_{x}\right)$ (with life time $\zeta=\infty)$ associated with $(B, V)$ in the resolvent sense is the reflecting Brownian motion on $\bar{D}$ (see [11, Example 4.5.3]).

Let $\tilde{v}$ denote the surface measure on $\partial D$. Then for $\tilde{v}$-a.e. $x \in \partial D$ there exists a unit inward normal vector $\mathbf{n}(x)=\left(\mathbf{n}_{1}(x), \ldots, \mathbf{n}_{d}(x)\right)$ (see [11, Example 5.2.2]). We consider the Neumann problems

$$
\partial_{s} u-\frac{1}{2} \Delta u+\lambda u=f(\cdot, u),\left.\quad \frac{\partial u}{\partial \mathbf{n}}\right|_{(0, \infty) \times \partial D}=g(x, u), \quad u(0, \cdot)=\varphi
$$

and

$$
-\frac{1}{2} \Delta v+\lambda v=f(\cdot, v),\left.\quad \frac{\partial v}{\partial \mathbf{n}}\right|_{\partial D}=g(\cdot, v),
$$


where $\frac{\partial u}{\partial \mathbf{n}}=\sum_{i=1}^{d} \mathbf{n}_{i} \frac{\partial u}{\partial x_{i}}$. It is known (see [11, Example 5.2.2]) that for every $x \in \bar{D}$ the process $X$ has under $P_{x}$ the representation

$$
X_{t}^{i}=X_{0}^{i}+B_{t}^{i}+\frac{1}{2} \int_{0}^{t} \mathbf{n}_{i}\left(X_{s}\right) d l_{s}, \quad t \geq 0, \quad P_{x} \text {-a.s. }
$$

where $B=\left(B^{1}, \ldots, B^{d}\right)$ is a $d$-dimensional standard Brownian motion and $l$ is the local time of $X$ on the boundary $\partial D$. Let $S_{00}(\bar{D})$ denote the set of all finite positive Borel measures $\gamma$ on $\bar{D}$ of finite energy integrals and such that $\left\|U_{1} \gamma\right\|<\infty$, where $U_{1} \gamma$ is the 1-potential of $\gamma$. It is also known (see [11, Example 5.2.2]) that $\tilde{v} \in S_{00}(\bar{D})$ and

$$
A^{0, \tilde{v}}=l .
$$

By Eqs. 6.14 and 6.15, the probabilistic solution of Eq. 6.12 (see [27, Section 4]) coincides with the probabilistic solution of

$$
\partial_{t} u-L u+\lambda u=f(x, u)+g(x, u) \cdot v, \quad u(0, \cdot)=\varphi
$$

with $v=d t \otimes \tilde{v}$, and the probabilistic solution of Eq. 6.13 (see [27, Section 5]) coincides with the probabilistic solution of

$$
-L w+\lambda w=f(x, w)+g(x, w) \cdot \tilde{v} .
$$

Proposition 6.5 Let $\varphi, f, g$ satisfy the assumptions of Theorem 4.3, and moreover, $f(\cdot, 0) \in$ $L^{1}(D ; m), g(\cdot, 0) \in L^{\infty}(D ; m)$. Let $u$ be a solution of Eq. 6.16 and $v$ be a solution of Eq. 6.17. Then for every $\lambda>0$ there is $C>0$ depending only on $d$ such that for q.e. $x \in \bar{D}$,

$$
\begin{aligned}
|u(t, x)-v(x)| \leq C e^{-\lambda t} t^{-d / 2}\left(\|\varphi\|_{L^{1}(D ; m)}+\lambda^{-1}\|f(\cdot, 0)\|_{L^{1}(D ; m)}\right. & \\
& \left.+\left(1 \vee \lambda^{-1}\right) m(D)\|g(\cdot, 0)\|_{\infty}\left\|R_{1}^{0} \tilde{v}\right\|_{\infty}\right), \quad t>0 .
\end{aligned}
$$

Proof By [2, Theorem 3.1] (see also [2, Lemma 4.3]), there is $C>0$ depending only on $d$ such that for every $\psi \in L^{1}(D ; m)$,

$$
\sup _{x \in \bar{D}} P_{t}^{0}|\psi|(x) \leq C t^{-d / 2}\|\psi\|_{L^{1}(D ; m)}, \quad t>0 .
$$

Moreover,

$$
\left\|R_{\lambda}^{0} f(\cdot, 0)\right\|_{L^{1}(D ; m)}=\left(f(\cdot, 0), R_{\lambda}^{0} 1\right)=\lambda^{-1}\|f(\cdot, 0)\|_{L^{1}(D ; m)} .
$$

Since $\tilde{v} \in S_{00}(\bar{D}),\left\|R_{1}^{0} \tilde{v}\right\|_{\infty}<\infty$. By the resolvent equation (see [11, Lemma 5.1.5]),

$$
R_{\lambda}^{0} \tilde{v}=R_{1}^{0} \tilde{v}+(1-\lambda) R_{\lambda}^{0}\left(R_{1}^{0} \tilde{v}\right)
$$

Hence $\left\|R_{\lambda}^{0}(g(\cdot, 0) \cdot \tilde{v})\right\|_{L^{1}(D ; m)} \leq\|g(\cdot, 0)\|_{\infty}\left\|R_{1}^{0} \tilde{v}\right\|_{L^{1}(D ; m)} \leq m(D)\|g(\cdot, 0)\|_{\infty}\left\|R_{1}^{0} \tilde{v}\right\|_{\infty}$ if $\lambda \geq 1$ and

$$
\begin{aligned}
\left\|R_{\lambda}^{0}(g(\cdot, 0) \tilde{v})\right\|_{L^{1}(D ; m)} \leq & m(D)\|g(\cdot, 0)\|_{\infty}\left(\left\|R_{1}^{0} \tilde{v}\right\|_{\infty}+(1-\lambda) \lambda^{-1}\left\|R_{1}^{0} \tilde{v}\right\|_{\infty}\right) \\
= & \lambda^{-1} m(D)\|g(\cdot, 0)\|_{\infty}\left\|R_{1}^{0} \tilde{v}\right\|_{\infty}
\end{aligned}
$$

if $\lambda<1$. The proposition follows immediately from the above estimates and Corollary 5.2. 


\subsection{Nonlocal Dirichlet forms}

Let $E=\mathbb{R}^{d}$ with $d \geq 2, m$ be the Lebesgue measure on $E$ and $\alpha \in(0,2)$. We consider the form

$$
B(u, v)=\int_{\mathbb{R}^{d}} \hat{u}(x) \hat{v}(x)|x|^{\alpha} d x, \quad u, v \in V,
$$

where $\hat{u}$ denotes the Fourier transform of $u$ and

$$
V=\left\{u \in L^{2}\left(\mathbb{R}^{d} ; m\right): \int_{\mathbb{R}^{d}}|\hat{u}(x)|^{2}|x|^{\alpha} d x<\infty\right\} .
$$

It is known that $(B, V)$ is a regular Dirichlet form on $L^{2}\left(\mathbb{R}^{d} ; m\right)$ (see [11, Example 1.4.1]). The operator $L$ associated with $(B, V)$ is the fractional Laplacian $\Delta^{\alpha / 2}$ and the Markov process $\mathbb{M}^{(0)}=\left(X, P_{x}\right)$ (with life time $\left.\zeta=\infty\right)$ associated with $(B, V)$ is a symmetric stable process of index $\alpha$.

Let $D \subset \mathbb{R}^{d}, d \geq 2$, be a nonempty open bounded connected set. Set $L_{D}^{2}\left(\mathbb{R}^{d} ; m\right)=\{u \in$ $L^{2}\left(\mathbb{R}^{d} ; m\right): u=0$ a.e. on $\left.D^{c}\right\}, V_{D}=\left\{u \in D(B): \tilde{u}=0\right.$ q.e. on $\left.D^{c}\right\}$, where $\tilde{u}$ is a quasicontinuous version of $u$. By [11, Theorem 4.4.3], the form $\left(B, V_{D}\right)$ is a regular Dirichlet form on $L_{D}^{2}\left(\mathbb{R}^{d} ; m\right)$, and by [11, Theorem 4.4.4], if $(B, V)$ is transient, then $\left(B, V_{D}\right)$ is transient, too.

Proposition 6.6 Let $\varphi, f, g$ satisfy the assumptions of Theorem 4.3, and moreover, $f(\cdot, 0) \in$ $L^{1}(D ; m), g(\cdot, 0) \cdot \tilde{\mu} \in \mathcal{M}_{0, b}(D)$. Let $u$ be a solution of Eq. 5.1 and $v$ be a solution of Eq. 5.2. Then there exists $C>0$ depending only on $d$, $\alpha$ such that q.e. $x \in D$,

$$
\begin{aligned}
|u(t, x)-v(x)| \leq C t^{-d / \alpha}\left(\|\varphi\|_{L^{1}(D ; m)}+(m(D))^{\alpha / d}\|f(\cdot, 0)\|_{L^{1}(D ; m)}\right. & \\
& \left.+(m(D))^{\alpha / d}(|g(\cdot, 0)| \cdot \tilde{\mu})(D)\right), \quad t>0 .
\end{aligned}
$$

Proof Let $\mathbb{M}_{D}^{(0)}$ denote the part of the process $\mathbb{M}^{(0)}$ on $D$ (see $[11$, Section 4.4$]$ ), $\zeta_{D}$ denote the life time of $\mathbb{M}_{D}^{(0)}$ and let $\left(P_{t}^{0}\right),\left(R_{\alpha}^{0}\right)$ denote the semigroup and the resolvent associated with $\mathbb{M}_{D}^{(0)}$. We denote by $p$ the transition density of the process $\mathbb{M}^{(0)}$. From the fact that $p(t, x, y)=p(t, 0, x-y)$ and the scaling property $p(t, 0, x)=t^{-d / \alpha} p\left(1,0, t^{-1 / \alpha} x\right)$ it follows that

$$
p(t, x, y) \leq C t^{-d / \alpha}, \quad t>0
$$

with $C=\sup _{x \in \mathbb{R}^{d}} p(1,0, x)$. Hence

$$
P_{t}^{0} \varphi(x) \leq C t^{-d / \alpha}\|\varphi\|_{L^{1}(D ; m)}, \quad t>0 .
$$

By Eq. 6.20 and [7, Theorem 1] (see also the proof of [8, Theorem 1.17]),

$$
\sup _{x \in D} E_{x} \zeta_{D}^{0} \leq c(m(D))^{\alpha / d}
$$

for some $c>0$ depending only on $\alpha, d$. By Eq. 6.21,

$$
P_{t}^{0}\left(R_{0}^{0}(|g(\cdot, 0)| \cdot \tilde{\mu})\right)(x) \leq C t^{-d / \alpha}\left\|R_{0}^{0}(|g(\cdot, 0)| \cdot \tilde{\mu})\right\|_{L^{1}(D ; m)} .
$$

Since

$$
\begin{aligned}
\left\|R_{0}^{0}(|g(\cdot, 0)| \cdot \tilde{\mu})\right\|_{L^{1}(D ; m)} & =\int_{D} R_{0}^{0} 1(x)|g(x, 0)| \tilde{\mu}(d x) \\
& =\int_{D} E_{x} \zeta_{D}^{0}|g(x, 0)| \tilde{\mu}(d x) \leq c(m(D))^{\alpha / d}(|g(\cdot, 0)| \cdot \tilde{\mu})(D),
\end{aligned}
$$


we have

$$
P_{t}^{0}\left(R_{0}^{0}(|g(\cdot, 0)| \cdot \tilde{\mu})\right)(x) \leq c(\alpha, d)(m(D))^{\alpha / d} t^{-d / \alpha}(|g(\cdot, 0)| \cdot \tilde{\mu})(D) .
$$

Putting $g=1$ and $\mu=f(\cdot, 0) \cdot m$ in the above estimate we get

$$
P_{t}^{0}\left(R_{0}^{0}|f(\cdot, 0)|\right)(x) \leq c(\alpha, d)(m(D))^{\alpha / d} t^{-d / \alpha}\|f(\cdot, 0)\|_{L^{1}(D ; m)} .
$$

Substituting (6.21)-(6.23) into (5.10) we get the desired estimate.

Assume additionally that $D$ has a $C^{1,1}$ boundary and $d \geq 3$. Then, by [19, Proposition 4.9], there exist constants $0<c_{1}<c_{2}$ depending only on $d, \alpha, D$ such that

$$
c_{1} \delta^{\alpha / 2}(x) \leq R_{0}^{0} 1(x) \leq c_{2} \delta^{\alpha / 2}(x), \quad x \in D,
$$

where $\delta(x)=\operatorname{dist}(x, \partial D)$. It follows that if

$$
\int_{D} \delta^{\alpha / 2}(x)|g(\cdot, 0)| \tilde{\mu}(d x)=: K<\infty
$$

then Eq. 6.23 holds with $|\mu(D)|$ replaced by $K$. Therefore under the above assumptions on $D$ the proof of Proposition 6.6 shows the following proposition.

Proposition 6.7 Let the assumptions of Proposition 4.1 hold, and moreover $f \in L^{1}(D ; m)$, $|g(\cdot, 0)| \cdot \tilde{\mu}$ satisfies (6.24). Then Eq. 6.19 holds true with $(m(D))^{\alpha / d}(|g(\cdot, 0)| \cdot \mu)(D)$ replaced by $K$.

Remark 6.8 (i) An analog of Proposition 6.6 holds true for $D$ as before and the form (6.18) replaced by any regular transient symmetric Dirichlet form $(B, V)$ on $L^{2}\left(\mathbb{R}^{d} ; d x\right)$ whose semigroup possesses a kernel $p$ satisfying uniform estimate of the form (6.20) with $\alpha / d$ replaced by $\kappa$, i.e.

$$
p(t, x, y) \leq C t^{-\kappa}, \quad t>0,
$$

for some $C, \kappa>0$. Indeed, an inspection of the proof of Proposition 6.6 shows that for such a form estimate (6.19) holds with $\alpha / d$ replaced by $\kappa$. A characterization of symmetric Dirichlet forms satisfying (6.25) in terms of Dirichlet form inequalities of Nash's type is given in [5]. For a concrete example of a class of forms satisfying (6.25) and containing the form (6.18) as a special case see [5, Remark 2.15]. For similar examples see [6, Examples $6.7 .14,6.7 .16]$.

\subsection{Local semi-Dirichlet forms}

Let $D \subset \mathbb{R}^{d}, m, H$ be as in Section 6.1, and let $a: D \rightarrow \mathbb{R}^{d} \otimes \mathbb{R}^{d}, b: D \rightarrow \mathbb{R}^{d}$ be measurable functions such that for every $x \in D$,

$$
\lambda^{-1}|\xi|^{2} \leq \sum_{i, j=1}^{d} a_{i j}(x) \xi_{i} \xi_{j} \leq \lambda|\xi|^{2}, \quad a_{i j}(x)=a_{j i}(x), \quad \sum_{i=1}^{d}\left|b_{i}(x)\right|^{2} \leq \lambda
$$

for some $\lambda \geq 1$. Set $V=H_{0}^{1}(D)$ and

$$
B(\varphi, \psi)=\sum_{i, j=1}^{d} \int_{D} a_{i j}(x) \frac{\partial \varphi}{\partial x_{i}} \frac{\partial \psi}{\partial x_{i}} d x+\sum_{i=1}^{d} \int_{D} b_{i}(x) \frac{\partial \varphi}{\partial x_{i}} \psi(x) d x, \quad \varphi, \psi \in V .
$$


Of course, the operator $L$ determined by $(B, V)$ has the form

$$
L=\sum_{i, j=1}^{d} \frac{\partial}{\partial x_{i}}\left(a_{i j}(x) \frac{\partial}{\partial x_{j}}\right)+\sum_{i=1}^{d} b_{i}(x) \frac{\partial}{\partial x_{i}} .
$$

By [26, Theorem 1.5.3], $(B, V)$ is a regular lower bounded semi-Dirichlet form on $H$. Let $G_{D}$ denote the Green function for $L$ on $D$ and $\bar{G}_{D}$ denote the Green function on $D$ for the Laplace operator $\Delta$. From Aronson's estimates (see [1]) it follows that there is $c>0$ such that $G_{D} \leq c \bar{G}_{D}$. Hence, if $\mu \in \mathcal{M}_{0, b}^{+}(D)$, then

$$
\begin{gathered}
\left(R_{0} \mu, 1\right)=\int_{D}\left(\int_{D} G_{D}(x, y) \mu(d y)\right) d x \leq c \int_{D}\left(\int_{D} \bar{G}_{D}(y, x) d x\right) \mu(d y) \\
\leq c\left\|\bar{G}_{D} 1\right\|_{\infty} \mu(D),
\end{gathered}
$$

which is bounded because $\left\|\bar{G}_{D} 1\right\|_{\infty} \leq c^{\prime}(m(D))^{2 / d}$ for some $c^{\prime}>0$ (see, e.g., [7, Theorem 1]). This shows that $\mathcal{M}_{0, b}^{+}(D) \subset \mathcal{R}^{+}(E)$. It is well known (see, e.g., [1]) that the transition density $p$ of the process associated with $(B, V)$ has the property that $p(t, x, y) \leq C t^{-d / 2}$, $t>0$, for some $C>0$, i.e. Eq. 6.20 with $\alpha=2$ is satisfied. Therefore there is an analog of Proposition 6.6 for equations involving the operator $L$ defined by Eq. 6.26.

Acknowledgments Research supported by Polish National Science Center (grant no. 2012/07/B/ST1/03508).

Open Access This article is distributed under the terms of the Creative Commons Attribution 4.0 International License (http://creativecommons.org/licenses/by/4.0/), which permits unrestricted use, distribution, and reproduction in any medium, provided you give appropriate credit to the original author(s) and the source, provide a link to the Creative Commons license, and indicate if changes were made.

\section{References}

1. Aronson, D.G.: Non-Negative Solutions of linear parabolic equations. Ann. Scuola Norm. Sup. Pisa 22, 607-693 (1968)

2. Bass, R.F., Hsu, P.: Some potential theory for reflecting Brownian motion in hölder and Lipschitz domains. Ann. Probab. 19, 486-508 (1991)

3. Boccardo, L., Gallouët, T.: Strongly nonlinear elliptic equations having natural growth terms and $l^{1}$ data. Nonlinear Anal. 19, 573-579 (1992)

4. Briand, P., Delyon, B., Hu, Y., Pardoux, E., Stoica, L.: $l^{p}$ solutions of backward stochastic differential equations. Stoch. Process Appl. 108, 109-129 (2003)

5. Carlen, E.A., Kusuoka, S., Stroock, D.W.: Upper bounds for symmetric Markov transition functions. Ann. Inst. H. Poincaré, Probab. Statist. 23(2, suppl.), 245-287 (1987)

6. Chen, Z.-Q., Fukushima, M.: Symmetric Markov processes, time change, and boundary theory. Princeton University Press, Princeton (2012)

7. Chung, K.L.: Greenian bounds for Markov processes. Potential Anal. 1, 83-92 (1992)

8. Chung, K.L., Zhao, Z.: From Brownian Motion to Schrödinger's Equation. Springer, Berlin Heidelberg (1995)

9. Dal Maso, G., Murat, F., Orsina, L., Prignet, A.: Renormalized solutions of elliptic equations with general measure data. Ann. Scuola Norm. Sup. Pisa Cl. Sci. (4) 28, 741-808 (1999)

10. Dellacherie, C., Meyer, P.-A.: Probabilités at Potentiel Chaptires V à VIII. Théorie des martingales. Revised edition. Hermann, Paris (1980)

11. Fukushima, M., Oshima, Y., Takeda, M.: Dirichlet Forms and Symmetric Markov Processes. Second Revised and Extended Edition. Walter de Gruyter, Berlin (2011)

12. Klimsiak, T.: Existence and large-time asymptotics for solutions of semilinear parabolic systems with measure data. J. Evol. Equ. 14, 913-947 (2014)

13. Klimsiak, T.: Semilinear elliptic systems with measure data. Ann. Mat. Pura Appl. (4) 194, 55-76 (2015) 
14. Klimsiak, T.: Semi-dirichlet forms, Feynman-Kac functionals and the Cauchy problem for semilinear parabolic equations. J. Funct. Anal. 268, 1205-1240 (2015)

15. Klimsiak, T., Rozkosz, A.: Dirichlet forms and semilinear elliptic equations with measure data. J. Funct. Anal. 265, 890-925 (2013)

16. Klimsiak, T., Rozkosz, A.: Obstacle problem for semilinear parabolic equations with measure data. J. Evol. Equ. 15, 457-491 (2015)

17. Klimsiak, T., Rozkosz, A.: Renormalized solutions of semilinear equations involving measure data and operator corresponding to Dirichlet form. NoDEA Nonlinear Diff. Equ. Appl. 22, 457-491 (2015)

18. Klimsiak, T., Rozkosz, A.: Semilinear elliptic equations with measure data and quasi-regular Dirichlet forms. Colloq. Math. 145, 35-67 (2016)

19. Kulczycki, T.: Properties of Green function of symmetric stable processes. Probab. Math. Statist. 17, 339-364 (1997)

20. Leonori, T., Petitta, F.: Asymptotic behavior for solutions of parabolic equations with natural growth terms and irregular data. Asymptot. Anal. 48, 219-233 (2006)

21. Liptser, R., Shiryayev, A.N.: Theory of Martingales. Nauka Moscow, 1986; English transl. Kluwer, Dordrecht (1989)

22. Ma, Z.-M., Röckner, M.: Introduction to the Theory of (Non-Symmetric) Dirichlet Forms. Springer, Berlin (1992)

23. Marcus, M., Véron, L.: Nonlinear Second Order Elliptic Equations Involving Measures. De Gruyter, Berlin (2014)

24. Murat, F., Porretta, A.: Stability properties, existence, and nonexistence of renormalized solutions for elliptic equations with measure data. Comm. Partial Diff. Equ. 27, 2267-2310 (2002)

25. Oshima, Y.: Some properties of Markov processes associated with time dependent Dirichlet forms. Osaka J. Math. 29, 103-127 (1992)

26. Oshima, Y.: Semi-Dirichlet Forms and Markov Processes. Walter de Gruyter, Berlin (2013)

27. Pardoux, E., Zhang, S.: Generalized BSDEs and nonlinear Neumann boundary value problems. Probab. Theory Relat. Fields 110, 535-558 (1998)

28. Petitta, F.: Asymptotic behavior of solutions for linear parabolic equations with general measure data. C. R. Math. Acad. Sci. Paris 344, 535-558 (2007)

29. Petitta, F.: Asymptotic behavior of solutions for parabolic operators of Leray-Lions type and measure data. Adv. Diff. Equ. 12, 867-891 (2007)

30. Petitta, F.: Large time behavior for solutions of nonlinear parabolic problems with sign-changing measure data. Electron. J. Differential Equations No. 132, pp. 10 (2008)

31. Petitta, F., Ponce, A.C., Porretta, A.: Diffuse measures and nonlinear parabolic equations. J. Evol. Equ. 11, 861-905 (2011)

32. Pierre, M.: Representant Precis d'Un Potentiel Parabolique, Seminaire de Theorie du Potentiel. Lect. Notes Math. 814, 186-228 (1980)

33. Porretta, A.: Existence results for nonlinear parabolic equations via strong convergence truncations. Ann. Mat. Pura Appl. (IV) 177, 143-172 (1999)

34. Protter, P. Stochastic Integration and Differential Equations, 2nd edn. Springer, Berlin (2004)

35. Stannat, W.: The Theory of Generalized Dirichlet Forms and Its Applications in Analysis and Stochastics, vol. 142 (1999) 